Canadian

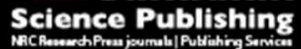

Canadian Journal of Forest Research Revue canadienne de recherche forestière

\title{
Random Forests and Stochastic Gradient Boosting for Predicting Tree Canopy Cover: Comparing Tuning Processes and Model Performance
}

\begin{tabular}{|r|l|}
\hline Journal: & Canadian Journal of Forest Research \\
\hline Manuscript ID & cjfr-2014-0562.R2 \\
\hline Manuscript Type: & Article \\
\hline Date Submitted by the Author: & 23-Jul-2015 \\
\hline Complete List of Authors: & $\begin{array}{l}\text { Freeman, Elizabeth; US Forest Service, Rocky Mountain Research Station } \\
\text { Moisen, Gretchen; US Forest Service, Rocky Mountain Research Station } \\
\text { Coulston, John; US Forest Service, Southern Research Station } \\
\text { Wilson, Barry; USDA, Forest Service }\end{array}$ \\
\hline Keyword: & $\begin{array}{l}\text { Tree canopy cover, Predictive mapping, Classification and Regression trees, } \\
\text { Stochastic gradient boosting, Random forest }\end{array}$ \\
\hline &
\end{tabular}




\title{
Random Forests and Stochastic Gradient Boosting for Predicting Tree Canopy Cover: Comparing Tuning Processes and Model Performance
}

\author{
Elizabeth A Freeman $^{\mathrm{a}, *}$, Gretchen G Moisen ${ }^{\mathrm{a}}$, John W. Coulston ${ }^{\mathrm{b}}$, and Barry T. Wilson ${ }^{\mathrm{c}}$ \\ ${ }^{a}$ USDA Forest Service, Rocky Mountain Research Station, 507 25th Street, Ogden, UT 84401, USA \\ ${ }^{\mathrm{b}}$ USDA Forest Service, Southern Research Station \\ ${ }^{\mathrm{c}}$ USDA Forest Service, Northern Research Station \\ *Corresponding author. Tel.: +1 801510 3756; E-mail address: eafreeman@fs.fed.us (E.A. Freeman)
}




\section{Abstract}

2 As part of the development of the 2011 National Land Cover Database (NLCD) tree canopy

3 cover layer, a pilot project was launched to test the use of high resolution photography coupled

4 with extensive ancillary data to map the distribution of tree canopy cover over four study regions

5 in the conterminous US. Two stochastic modeling techniques, Random Forests (RF) and

6 Stochastic Gradient Boosting (SGB), are compared. The objectives of this study were first to

7 explore the sensitivity of RF and SGB to choices in tuning parameters. Second, to compare the

8 performance of the two final models by assessing the importance of, and interaction between,

9 predictor variables, the global accuracy metrics derived from an independent test set, as well as

10 the visual quality of the resultant maps of tree canopy cover. The predictive accuracy of RF and

11 SGB was remarkably similar on all four of our pilot regions. In all four study regions, the

12 independent test set MSE was identical to 3 decimal places, with the largest difference in Kansas

13 where RF gave an MSE of 0.0113 and SGB gave an MSE of 0.0117. With correlated predictor

14 variables, stochastic gradient boosting had a tendency to concentrate variable importance in

15 fewer variables, while Random Forest tended to spread importance out amongst more variables.

$16 \mathrm{RF}$ is simpler to implement than SGB, as RF both has fewer parameters needing tuning, and also

17 was less sensitive to these parameters. As stochastic techniques, both RF and SGB introduce a

18 new component of uncertainty: repeated model runs will potentially result in different final

19 predictions. We demonstrate how RF allows the production of a spatially explicit map of this

20 stochastic uncertainty of the final model.

21 Key Words: Tree canopy cover; Predictive mapping; Classification and Regression trees;

22 Random forest; Stochastic gradient boosting 
23

41 Nationwide mapping of tree canopy cover posed numerous technical questions: alternative 42 means to observe tree canopy cover (Frescino and Moisen 2012), the relationship between photo-

43 based tree canopy cover and canopy modeled from extant forest inventory plots (Toney et al. 44 2012), repeatability in photo-interpretation (Jackson et al. 2012), efficient sampling strategies

\section{Random Forests and Stochastic Gradient Boosting for Predicting Tree Canopy Cover: Comparing Tuning Processes and Model Performance}

\section{Introduction}

The tree canopy cover in a given area is a primary structural characteristic of both forested and non-forested landscapes. Understanding and quantifying the spatial distribution of tree canopy cover is relevant to many applications, including forest management (Jennings et al. 1999), fire modeling (Rollins and Frame 2006), air pollution mitigation (Nowak et al. 2006), stream and water temperature (Webb and Crisp 2006), and carbon storage (Kellndorfer et al. 2006). Because of the importance of tree canopy cover, Homer et al. (2007) developed a $30 \mathrm{~m}$ geospatial dataset of percent tree canopy cover in the United States as part of the 2001 National Land Cover Database (NLCD, http://www.mrlc.gov/). As part of the development of the updated 2011 NLCD tree canopy cover layer, a national pilot project was launched to test the use of high resolution photography acquired though the National Agriculture Imagery Program (NAIP) coupled with extensive ancillary data layers through alternative sampling and modeling methodologies (Coulston et al. 2012). 
45 (Tipton et al. 2012), and choice of appropriate subpopulations over which to construct predictive

46 models (Moisen et al. 2012). Related to modeling methodologies, Coulston et al. (2012)

47 compared a parametric modeling technique, beta regression, with the non-parametric technique

48 of random forests and found that the random forest modeling technique outperformed the beta

49 regression approach, based on pseudo $\mathrm{R}^{2}$, root mean squared error, and slope verses intercept of

50 the observed verses predicted regression line. In addition, they found that the degree to which

51 Random Forest outperformed beta regression was related to model quality. In regions with high

52 quality models RF only slightly outperformed beta regression, while in regions with low quality

53 models RF offered a more marked improvement. Now, in this study, two non-parametric

54 modeling techniques, both involving an ensemble of classification and regression trees, are

55 compared: Random Forests (RF) and Stochastic Gradient Boosting (SGB).

56 There has been little direct comparison of Random Forest and Stochastic Gradient Boosting for

57 continuous response models, though previous work by Chirici, et al. (2012) looking at

58 categorical response models found that SGB out performed RF, particularly for the rare

59 categories.

60 RF (Breiman 2001) has received considerable attention in the ecological literature. Prasad et al.

61 (2006) gave an overview of the use of Random Forests for ecological prediction. Baccini et al.

62 (2008) used RF to map above ground biomass in Tropical Africa from satellite imagery. Chan

63 and Paelinckx (2008) compared RF to Adaboost for mapping ecotopes from airborne

64 hyperspectral imagery and found both models to work well, with RF having the advantage of

65 faster training time and greater stability and robustness while Adaboost had a slight advantage in

66 accuracy. Evans and Cushman (2009) used RF to produce continuous probability of occurrence 
67 maps for four conifer species in Northern Idaho, USA, based on a combination of climactic, 68 topographic and spectral predictor variables. Gislason et al. (2006) found that RF performed well

69 compared to basic Classification and Regression Tree (CART) models, as well as other bagging

70 and boosting models, for landcover classification based on Landsat and topographic predictors.

71 Lawrence et al. (2006) found that RF outperformed CART models for mapping invasive plants

72 using hyperspectral imagery, and also found that the Out-of-Bag accuracy assessments provided

73 by RF were reliable when compared to withheld test data. Powell et al. (2010) compared RF to

74 Reduced Major Axis regression and Gradient Nearest Neighbor imputation for mapping biomass

75 from Landsat satellite imagery and found that RF performed favorably in terms of RMSE,

76 though poorly in terms of variance ratio.

77 SGB (Friedman et al. 2000; Friedman 2001; Friedman 2002) is now gaining recognition in

78 ecological modeling. De'ath (2007) summarized the benefits SGB can offer ecologists and

79 provides an introduction to its use with ecological datasets, while Elith et al. (2008) provided

80 guidelines for its use in ecological modeling. However, little attention has been paid to tuning

81 SGB models in ecological applications. For example, Güneralp, et al. (2014) and Filippi et al.

82 (2014) compare SGB, multivariate adaptive regression splines (MARS), and Cubist for mapping

83 aboveground floodplain biomass and found that both SGB and MARS outperformed Cubist.

84 Both papers examined mapping at a relatively small spatial scale - their map area was a single

85 river bend of $0.219 \mathrm{~km}^{2}$. Their general results may be applicable at all scales, but they did not

86 look at the practical issues of tuning SGB models for large scale maps. Güneralp, et al. (2014)

87 sets tuning parameters empirically, while Filippi et al. (2014) does not examine tuning in detail

88 but states that they tested other learning rates, and found poorer performance. 
89 Leathwick, et al. (2006) found SGB to offer superior predictive performance to Generalized

90 Additive Models (GAM) for predicting and mapping fish species richness. They looked at three

91 values for the interaction depth parameter, and left the other parameters at their default values.

92 Sankaran et al. (2008) used SGB not to produce maps, but rather to investigate the relationship

93 between predictor variables in regulating the woody cover in African savannas. They used the

94 default shrinkage parameter, and used cross validation to determine optimal values of interaction

95 depth and number of trees. Lawrence et al. (2004) compared SGB and Classification Tree

96 Analysis (CTA) for classification of remotely sensed imagery for use in forestry and found that

97 SGB improved accuracy, though the strength of the improvement was dataset dependent. They

98 do not descript how their SGB parameters were set. Moisen et al. (2006) compared the

99 performance of SGB, GAM, and proprietary tree-based methods for predicting tree species

100 presence and basal area in Utah and found that for the majority of species, SGB models were the

101 most accurate at predicting species presence, and competitive with the other techniques for

102 predicting basal area. Parameters were set based on a related dataset, but the tuning process is not

103 described. Baker et al. (2006) found SGB more effective than CTA for mapping wetlands and

104 riparian areas from Landsat data supplemented with topographic and soils data, though model

105 parameter selection is not mentioned. Pittman et al. (2009) found that SGB worked well to

106 predict the diversity and abundance of fish and corals from underwater Lidar Bathymetry with

107 parameters left at their default values.

108 In addition to the issues of model tuning, stochastic techniques such as RF and SGB also

109 introduce a new component of uncertainty: repeated model runs will potentially result in

110 different final predictions. This uncertainty can be reduced by careful model tuning. And, in the

111 case of RF models, it is possible to produce a spatially explicit map based estimate of the 
112 stochastic uncertainty remaining in the final model. In RF the individual trees are independent,

113 allowing the standard deviation of the individual tree predictions to be calculated for each pixel

114 in the map, providing a measure of the stochastic variability in the map's predictions.

115 Stochastic models such as RF and SGB are powerful modelling techniques that have been shown 116 to provide superior predictive performance to parametric methods in a variety of applications

117 (e.g. Moisen and Frescino 2002, Prasad et al.2006, Powell et al. 2010) as well as for the specific

118 datasets examined here (Coulston et al. 2012). RF has been used extensively in ecological and

119 remote sensing applications, while SGB is beginning to gain recognition. However, there has

120 been little direct comparison of these two techniques for continuous ecological data, which this

121 paper provides. In addition, the non-parametric modeling literature calls attention to the

122 importance of tuning in the modeling process. Yet, in the applied ecological and natural resource

123 literature, particularly predictive modeling of forest attributes, this important phase of the

124 modeling process is often ignored. Consequently, we explore the sensitivity of RF and SGB to

125 choices in tuning parameters in modeling tree canopy cover over four diverse study regions in

126 the conterminous US. Second, the study sought to compare the performance of the two final

127 models in each study area by assessing the importance of, and interaction between, predictor

128 variables under each modeling technique, the global accuracy metrics derived from an

129 independent test set, as well as the visual quality of the resultant maps of tree canopy cover.

130 Finally, for the RF model we produce a map of the stochastic uncertainty remaining in the final

131 tuned model, and illustrate the value of this metric for map users by examining in detail two

132 areas of this map that show particularly high levels of uncertainty.

\section{2. Materials and Methods}




\section{$134 \quad 2.1$ Study Regions}

135 Four study regions in the United States were used in this pilot study, located in Georgia (GA), 136 Kansas (KS), Oregon (OR), and Utah (UT). These locations provide a contrast between high

137 crown cover (GA and OR) and lower crown cover (KS and UT). Kansas in particular posed a

138 challenge to model tuning as over half the study region has zero tree canopy cover. These

139 locations also provide a contrast in the types of tree cover present. Georgia has a higher

140 proportion of broad leaf tree species, while Oregon has a high proportion of conifer spieces. Utah

141 includes large areas of woodland pinyon-juniper forests (Table 1).

142 Each study region was approximately the size of one Landsat Scene, and they were selected to

143 cross local ecological gradients. For example, the GA study area ranged from the Piedmont

144 region in the south, through the Atlanta metropolitan area, to the heavily forested Appalachian

145 Mountains in the north.

\section{$146 \quad$ 2.2 Data}

147 An intensive (approximately $1 \mathrm{~km} \mathrm{x} 1 \mathrm{~km}$ ) grid of photo interpretation plots was established over 148 the four pilot regions. The imagery is provided by the National Agriculture Imagery Program 149 (NAIP) (USDA 2009) collected during the growing season in 2009. The grid for locating the 150 photo plots is adopted from the FIA sample design (Bechtold and Patterson, 2005) of a quasi151 systematic sample based on White, et al. (1992). This design is assumed to produce a random 152 equal probability sample (McRoberts, et al., 2006). The FIA sample design has a nominal 153 sampling intensity of approximately one sample location per 2,400ha across all land covers and 154 types. For this pilot study the FIA sample design has been intensified 4X intensity (1 plot per 600 155 ha) as described by White, et al. (1992). 
156 Each photo plot consisted of a 105 point triangular-grid distributed in a $90 \mathrm{~m}$ x $90 \mathrm{~m}$ square area

157 surrounding the sample location. Each dot was characterized as "tree canopy" or "no tree

158 canopy". The response variable of percent tree canopy cover was defined as the proportion of

159 tree canopy dots identified on the photo plot. The design based estimators of proportion canopy

160 cover in each photo plot, mean proportion canopy cover in each study region, and standard error

161 of the estimate were obtained following Cochran (1977) (Table 2).

162 Predictor variables included transformed aspect, slope, elevation, topographic positional index

163 (Moore 1991), Bailey's ecoregions (Bailey 1995), land cover and tree canopy cover from the

1642001 NLCD (Homer et al. 2004), and Landsat-5 reflectance bands (

165 Table 3). The Landsat data were also leaf-on and from either 2008 or 2009, depending on cloud

166 cover. Because many of the predictor variables originated from $30 \mathrm{~m}$ pixel resolution products,

167 assignment to each $90 \mathrm{~m}$ x $90 \mathrm{~m}$ plot was accomplished by taking a focal mean over a 3 pixel x 3

168 pixel window for continuous variables, and a focal majority for the categorical variables. See

169 Coulston et al. (2012) for more details on data used in this study.

170 This study is part of a larger mapping project whose goal is updating and improving the 171 2001National Landcover Database. This drove the selection of a number of the predictor

172 variables used in this study.

173 Because we have relatively large data sets (approximately 4,000 data points per region), we were 174 able to use independent tuning and test data to build and evaluate both RF and SGB models. We 175 randomly assigned $25 \%$ of the data from each region as a tuning set and $25 \%$ as independent test 176 data, leaving 50\% for model training, as suggested by Hastie et al. (2009). 


\section{$177 \quad 2.3$ Models}

178 CART models (Breiman et al. 1984) are flexible and robust tools that are well suited to the task 179 of modeling the relationship between a response and a set of explanatory variables for the 180 purposes of making spatial predictions in the form of a map. These are intuitive methods, often 181 described in graphical or biological terms. A CART model begins at its root. An observation 182 passes down the tree through a series of splits, or nodes, at which a decision is made as to which

183 direction to proceed based on values of the explanatory variables. Ultimately, a terminal node, or 184 leaf, is reached and a predicted response is given, which is typically the mean of observations in 185 the terminal node for a continuous response, or a plurality vote for a categorical response. See 186 De'ath and Fabricius (2000) for a thorough explanation and Moisen (2008) for a simple 187 overview.

188 Although CART models are powerful tools by themselves, much work has been done in the data

189 mining and machine learning fields to improve the predictive ability of these models by 190 combining separate tree models into what is often called a committee of experts, or ensemble.

191 Two such ensemble techniques considered here are RF and SGB models.

192 As discussed earlier, RF is receiving increasing attention in the ecological and remote sensing

193 literature. In this technique, a bootstrap sample of the training data is chosen. At the root node, a 194 small random sample of explanatory variables is selected and the best split made using that 195 limited set of variables. At each subsequent node, another small random sample of the 196 explanatory variables is chosen, and the best split is made. The tree continues to be grown in this 197 fashion until it reaches the largest possible size, and is left un-pruned. The whole process, 198 starting with a new bootstrap sample, is repeated 500 or more times. The final prediction is a 
200 the trees in the collection. Because each tree is built from a subsample of the training data, the

201 un-sampled portion of the data can be used to produce Out-Of-Bag (OOB) model predictions for

202 that tree. In addition, these independent trees allow a pixel by pixel estimate of the variability in

203 the predictions of the individual trees in the final model. The standard deviation of these

204 predictions can be calculated for each pixel. This is not to be misconstrued as a prediction

205 interval, but instead is a useful measure of uncertainty in the resultant maps.

206 Unlike RF, which is an ensemble of independent trees, SGB sequentially builds many small

207 classification or regression trees sequentially from "pseudo"-residuals (the gradient of the loss

208 function) of the previous tree. At each iteration, a tree is built from a random sub-sample of the

209 "pseudo"- residuals (selected without replacement) producing an incremental improvement in the

210 model. In SGB the trees are not grown to completion (as in RF); instead, the maximum tree size

211 is specified by a model parameter.

\section{$212 \quad 2.4$ Software}

213 Analysis was conducted in the R software environment (R Development Core Team, 2008)

214 using the package ModelMap (Freeman and Frescino 2009). Many of the diagnostic and

215 graphical tools available in the current version of this package were developed concurrently with

216 this study to address questions that occurred while comparing these models. ModelMap

217 constructs predictive models of continuous or discrete responses by calling the R packages

218 randomForest (Liaw and Weiner 2002) and gbm (Ridgeway et al. 2013), respectively. These

219 models are then applied to image files of predictors to create detailed prediction surfaces.

\subsection{Tuning Process}




\subsubsection{Tuning Random Forest}

$222 \mathrm{RF}$, as implemented by the $\mathrm{R}$ package randomForest, only requires the user to make decisions

223 about two tuning parameters. The first, mtry, controls the number of predictor variables

224 randomly sampled to determine each split. RF models are relatively insensitive to the choice of 225 mtry (Breiman 2001, Liaw and Weiner 2002), though higher values of mtry tend to work better

226 in cases where only a few of the predictors contribute to the model, and there are a lot of "noise"

227 predictors containing no useful information (Liaw and Weiner 2002, Prasad et al.2006). The

228 second tuning parameter, ntrees, controls the total number of independent trees. The number of

229 trees required to stabilize variable importance (Liaw and Weiner 2002) and variable interaction

230 (Evans and Cushman 2009, referencing personal communication of Adele Cutler) may be larger

231 than the number required to stabilize prediction accuracy.

232 For mtry, a suggested starting point for tuning continuous response models is the number of

233 predictor variables divided by three, followed by checking half this number and twice this

234 number. Our models had 14 predictor variables, and thus we considered 3 possible values for

235 mtry: 2, 4, and 8 . Using the 50\% training data set, we built 20 models for each value of mtry,

236 with 2,500 trees each.

237 From each model, predictions were made on the $25 \%$ tuning data set from subsets of increasing 238 numbers of trees (100 trees, 200 trees... 2500 trees). Three error measures (mean squared error 239 (MSE), Pearson's correlation, and Spearman's correlation) were plotted against number of trees, 240 with the mtry value of each model indicated by line color.

\subsubsection{Tuning Stochastic Gradient Boosting}


242 SGB, implemented by the $\mathrm{R}$ package $\mathbf{g b m}$ (Ridgeway et al. 2013) requires the user to make

243 choices about a larger number of tuning parameters, including shrinkage, bagging fraction,

244 interaction depth, and number of trees. Note that the names used for these parameters differ

245 throughout the literature. This is just differing terminology for describing the same parameters,

246 but these differing terminologies can be daunting for new users. Even the name of the technique

247 itself varies in the literature. Table 5 provides a cross reference for translating amongst the 248 terminologies used by different authors.

249 Tuning SGB models is complicated by the fact that changing any one of the parameters can

250 affect the optimal values of the other parameters. Shrinkage, also known as the learning rate, 251 controls the influence of each successive tree on the final predictions. A lower shrinkage (i.e., a 252 slower learning rate) increases the number of trees required and thus increases computing time, 253 but also reduces the chance of over fitting. Bagging fraction, also known as sampling fraction, 254 controls the fraction of the training data randomly selected to build each tree. Smaller bagging 255 fractions reduce the chance of over fitting, but result in increased variability between model runs, 256 i.e. increased model uncertainty (Friedman 2002). Interaction depth, also known as tree size or 257 tree complexity, controls the maximum size of each tree. As in RF, number of trees controls the 258 total number of trees. Unlike in RF, using too many trees in SGB does result in overfitting and 259 poorer model performance on independent test data. Ridgeway (2007) recommended balancing 260 shrinkage and number of trees to result in models with between 3,000 and 10,000 trees and 261 shrinkage rates between 0.01 and 0.001 . Elith et al. (2008) recommended models have at least 2621,000 trees. 
263 As in RF, we built models using the 50\% training data, and then used accuracy statistics

264 calculated on the $25 \%$ tuning data to optimize model parameters. We started by building 10

265 models of 6,000 trees each, for combinations of shrinkage $(0.008,0.004,0.002,0.001)$, bagging

266 fraction (0.1 to 0.9$)$, and interaction depth $(1,2,4,8)$, for each of the four regions. We calculated

267 the three error measures (MSE, Pearson's correlation, and Spearman's correlation) using the

268 tuning data, and then averaged these error measures over the 10 models for each combination of

269 model parameters. We then followed this with a separate fine-tuning for each individual region.

270 For example, in pilot regions that seemed to still be improving at an interaction depth of 8 , we

271 tried interaction depths of 10 and 12. After optimizing bagging fraction and interaction depth, we

272 selected a shrinkage that resulted in a model that met our goal of having the optimum number of

273 trees lie between 3,000 and 5,000, to balance model accuracy with reasonable computational

274 efficiency.

\section{$275 \quad 2.6$ Model Comparisons}

276 One final RF and one final SGB model were run for each of the four pilot regions using the 50\%

277 training data and tuning parameters optimized through the tuning process described above.

278 Comparisons were then made in each pilot area between the two final models by assessing the

279 predictor variables for relative variable importance and interaction effects under each modeling

280 technique, the map accuracy metrics derived from the $25 \%$ test set, as well as the quality of the

281 resultant maps of tree canopy cover.

\section{$282 \quad$ 2.6.1 Role of Predictor Variables}


283 Unlike simpler model structures such as linear models, tree based ensemble models do not have a

284 straight-forward formula linking predictor variables to model predictions. There are, however,

285 several techniques that can be used to shed some light on the underlying relationships.

286 Variable Importance - Both RF and SGB provide estimates of the relative importance of each

287 predictor variable to the final model. The randomForest package offers two options for

288 calculating variable importance, first by permuting OOB data, and second by calculating the

289 decrease in node impurities from splitting on the variable. The gbm package calculates the

290 relative influence of each variable in reducing the loss function. With continuous response

291 (modeled with a Gaussian loss function), this is the reduction of squared error attributable to

292 each variable. For this study we compare the permutation importance for our RF models with the

293 relative influence of our SGB models.

294 Variable Interactions - We also examined two-way interactions between predictor variables

295 graphically using the three dimensional partial dependency plots presented in Elith et al. (2008).

296 The 'model.interaction.plot' function from the ModelMap package further developes these plots

297 to include the ability to investigate both continuous and categorical predictor variables. In these

298 plots, the predictor variables are examined two at a time. An x-y grid is created of possible

299 combinations of predictor values over the range of both variables. The remaining predictor

300 variables are fixed at either their means (for continuous predictors) or their most common value

301 (for categorical predictors). Model predictions are generated over this grid and plotted as the $\mathrm{z}$ 302 axis.

\section{$303 \quad$ 2.6.2 Accuracy Measures}


304 Histograms were made of the distribution of Tree Canopy Cover (TCC) for the observed test

305 data within each pilot area, and compared to the histograms of the RF and the SGB predictions.

306 In these histograms the vertical bars represent the number of photo plots in the test set that have a

307 given value of percent canopy cover. Comparing the observed distribution of plots as a function

308 of crown cover to that of the model predictions, allows an assessment of how well the model

309 predictions capture the range of the observed data. In particular, how well they predict the

310 extremes of the data - does each model predict an accurate proportion of very low and very high

311 canopy cover?

312 In addition, for each pilot area, we examined final model accuracy in terms of MSE, Pearson's

313 and Spearman's correlation coefficients, the difference between the mean of the observed TCC

314 and the mean of the predicted TCC, and the slope and intercept of the observed versus predicted

315 TCC regression line. Pearson's correlation coefficient is a measure of the linear relationship,

316 while Spearman's correlation coefficient is a measure of the monotonic relationship (Hauke,

317 2011). While it is common to use Pearson's correlation for ordinal data and Spearman's for

318 ranked data, Spearman's can also be useful even for ordinal data if there are outliers, or to

319 identify a non-linear but monotonic relationship.

\section{$320 \quad$ 2.6.3 Map Quality}

321 We used the final RF and SGB model for Utah to produce maps for that region, with predictions

322 for each $90 \mathrm{~m}$ pixel. The maps were generated using the same scales and colors, and examined

323 visually for spatial structures that differed between the RF and SGB predictions. Histograms of

324 number of pixels predicted by percent predicted TCC over all map pixels in the Utah pilot region 325 were compared for the final RF and SGB models. 
326 A map of the stochastic uncertainty remaining in the final RF model for Utah was also created.

327 This map was built by calculating the standard deviation for each pixel from the predictions of

328 each of the independent randomly generated trees that compose the RF model. If the individual

329 trees are in agreement the uncertainty is low. If the trees are not in agreement, with some trees

330 predicting low TCC while others predict high TCC, then the uncertainty is higher. In SGB, the

331 trees are not independent, thus this uncertainty map is not available.

\section{3. Results}

\section{3.1 Tuning Process}

\section{$334 \quad$ 3.1.1 Tuning Random Forest Models}

335 As suggested by Breiman (2001) and Liaw and Weiner (2002), random forest proved to be

336 relatively insensitive to the choice of mtry (Figure 1). Figure 1 illustrates how accuracy metrics

337 are affected by the ntree and mtry parameters for two of our study regions. The lines represent

338 each independent model run, with the shade of the lines indicating the value of mtry used for that

339 particular model run. The vertical spread of a given color indicated the variation between

340 independent model runs, in other words, the stochastic uncertainty. This uncertainty is highest

341 when the models have relatively few trees, and decreases as more trees are added. The influence

342 of mtry can be seen by the distance between the three shades of the lines once the model runs

343 have stabilized at higher values of ntree. In this figure Georgia shows greater stochastic

344 uncertainty for a given value of the mtry parameter (a greater spread within the lines of a given

345 color), particularly for low values of ntree, while Kansas shows a slightly greater sensitivity to 
346 the mtry parameter (a greater distance between the three colors), particularly at higher values of 347 ntree.

348 In most regions MSE and correlation stabilized at between 1000 and 1500 trees, and there was

349 no evidence of over fitting with the larger number of trees. As trees are added to the models both

350 the training and the tuning MSE initially decrease, then stabilize at higher numbers of trees

351 (over fitting would have led to the tuning data MSE increasing with higher numbers of trees

352 while the training MSE continued to decrease). Keeping in mind that stabilizing variable

353 importance (addressed later) may require more trees than stabilizing model accuracy (Liaw and

354 Weiner 2002), we used 2000 trees for our final models. The four study regions exhibited similar

355 effects. The RF models were relatively insensitive to the mtry parameter. Of the four study

356 regions, Kansas showed the greatest sensitivity to mtry, but even in that region the effects of

357 varying mtry were minor: When the tuning set error statistics were compared for the three values

358 of mtry MSE ranged from 0.014 to 0.015 ; Pearson's correlation ranged from 0.868 to 0.865 ;

359 Spearman's correlation ranged from 0.63 to 0.64 ; and, the predicted mean ranged from 0.129 to

$360 \quad 0.130$. Overall, Georgia and Oregon performed very slightly better with mtry of 4 and Kansas

361 and Utah with mtry of 8.

\section{$362 \quad$ 3.1.2 Tuning Stochastic Gradient Boosting Models}

363 For each value of shrinkage we plotted the average error measures (from the optimal number of

364 trees for that combination of parameters) as a function of bagging fraction, with interaction depth

365 represented by point size (Figure 2). Better models have lower MSE and higher correlation.

366 The SGB models do show overfitting for higher numbers of trees, and the number of trees that

367 leads to overfitting varies with the values of the other parameters. The tuning error initially 
368 decreases as trees are added, but then as more trees are added the tuning error begins to rise 369 again. The number of trees used in the final SGB models was chosen to minimize this over 370 fitting.

371 With larger values of shrinkage (shrinkage $=0.05$ and 0.01 ) models begin to over fit before

372 reaching optimum model accuracy (Figure 3). However, we found that for the smaller values of

373 shrinkage (shrinkage $\leq 0.005$ ) the models all reached similar accuracy, before over fitting was

374 observed. For these smaller shrinkage values we found the relationship between model quality,

375 bagging fraction and interaction depth was generally stable as shrinkage was changed, given that

376 the number of trees was increased to compensate for the lower shrinkage (slower learning rate).

377 It just took more trees to reach optimal model accuracy at lower (slower) shrinkages (Figure 3).

378 Therefore we began by picking the optimal combination of bagging fraction and interaction

379 depth, and then selected the shrinkage that resulted in 3000-5000 trees. Notice that the same

380 shrinkage rate requires more trees to reach the best model performance in Georgia than in

381 Kansas (Figure 3). Our goal of 3000-5000 trees required a shrinkage rate of 0.001 in Kansas,

382 while Georgia, Oregon and Utah required the slightly faster shrinkage rate of 0.002 to have the

383 final number of trees in the preferred range.

384 We expected to find that the best models had bagging fractions near 0.5. Ridgeway (2007)

385 suggests starting with bagging fractions near 0.5, Elith et al. (2008) found bagging fractions

386 between 0.5 and 0.75 worked best. Friedman's data (2002) was best modeled with a bagging

387 fraction of 0.4 . In some of our regions bagging fractions near 0.5 did give the best results. For

388 example in Georgia, the test MSE and the Pearson's correlation were best at bagging

389 fraction=0.4, while Spearman's correlation was optimized at a slightly higher bagging fraction $=$ 
$390 \quad 0.6$ (Figure 3). And Utah performed best with a bagging fraction of 0.5. In Oregon bagging

391 fraction had little effect on Model quality, but a quite low bagging faction $=0.2$ seemed to very

392 slightly improve model fit. Smaller bagging fractions introduce more stochasticity into the

393 model, and therefore it can counter act over fitting (Friedman 2002). This increased stochasticity

394 can be observed in Figure 3, where the lines representing very low bagging fraction show a jitter.

395 In contrast, Kansas (where 53\% of plots have zero TCC) did better with a larger bagging fraction

396 of 0.7 (Figure 3 ). This is probably due to the fact that with a higher bagging fraction of 0.7 , it is

397 more likely that each tree with be based on a subset containing at least some non-zero responses.

398 To balance computational complexity with model improvement, we settled on interaction depth

399 of 10 for the 4 regions. Some of our regions showed slight improvement with even higher

400 interaction depths, but we seemed to be reaching a point of diminishing returns. For example,

401 increasing interaction depth form 10 to 12 had much less of an effect than the change from 8 to

402 10. An interaction depth of 10 was a compromise between model improvement, computation

403 time, and risk of over fitting. We then selected a shrinkage rate slow enough to maintain the

404 desired 3000-5000 trees. The numbers of trees for the final models ranged between 3500 and 4054500.

\section{$406 \quad 3.2$ Role of Predictor Variables}

\section{$407 \quad 3.2 .1 \quad$ Predictor Variable Importance}

408 We expected the most important predictors to be similar between the SGB and the RF models.

409 This proved true in two of our regions (Kansas and Utah), but in the other two regions (Georgia 410 and Oregon) RF and SGB differed in their choice of most important variable (Figure 4). In both

411 of these regions the most important SGB predictor was a remote sensing band (band 3 in 
412 Georgia, band 6 in Oregon). These remote sensing bands were highly correlated with several of

413 the other bands (between band correlation up to 0.92 in Georgia and 0.93 in Oregon) and

414 moderately negatively correlated with TCC2001 (correlation with TCC2001 of -0.55 in Georgia

415 and -0.75 in Oregon). RF models spread the importance amongst the correlated predictors, while

416 SGB models concentrated the importance in a single band.

417 For both RF and SGB models, most of the variable importance is split between two predictors in

418 Kansas. Unlike SGB importance, in RF when the importance is concentrated in a small number

419 of variables, it does suggest that the other predictors are noise variables. In such cases, using a

420 higher value of mtry can often improve the model, as it is more likely that the randomly selected

421 variables for each split will contain at least one non noise variable. This may explain why

422 Kansas was the pilot region that performed better with a higher than default value for mtry (mtry

$423=8$ instead of mtry $=4)$.

\section{$424 \quad$ 3.2.2 Predictor Variable Interactions}

425 We did not find dramatic interaction effects in this dataset, but we did find subtle interactions.

426 For example, in the RF model for Utah there are some interactions between Land Cover class

427 and elevation (Figure 5), in particular there are some interesting difference in the effect of

428 elevation in Land Cover classes 41 (Deciduous Forest) and 42 (Evergreen Forest). This figure

429 shows the predicted TCC for each Land Cover class across the range of elevations found in the

430 training dataset for UT. In Deciduous Forest, TCC is moderate at the lowest elevations, increases

431 at mid elevations, and drops slightly to moderate levels at high elevations. In class Evergreen

432 Forest, TCC starts slightly higher than class 41 at low elevations, rises at mid elevations, but then 433 drops to near zero at high elevations. 
434 Also, in Figure 5 notice the differences between the RF model and the SGB model. Even though

435 overall accuracy of the two models is similar, the interaction plots highlight subtle differences in

436 the relations ships of TCC to the predictor variables. For example, while both RF and SGB

437 models show a peak crown cover at low to mid elevations (1600m to $2400 \mathrm{~m})$, the SGB model

438 also has a small bump in crown cover at higher elevation $(3000 \mathrm{~m})$.

439 Another example of differing predictor effects in Utah is illustrated in Figure 5 by the predicted

440 TCC in Land Cover Class 11 (open water). The SGB model is predicting high TCC for points

441 landing in open water in Utah, particularly at low to mid elevations, though the difference can be

442 seen to some extent at all elevations. A possible explanation may be seen in the variable

443 importance plot for Utah (Figure 4). The SGB model concentrated importance in TCC2001,

444 while the RF model spread the importance between multiple predictors.

445 The interaction plots examine the effects of the two selected predictors with the remaining

446 variables fixed at their mean value (or most common value for categorical predictors). Therefore

447 Figure 5 shows the theoretical predictions for pixels at each land cover class and elevation with 448 average values of TCC2001 (and all other predictors.) In the training data, all points with Land

449 Cover Class 11 have TCC2001 of zero, whereas the overall mean value of TCC2001 is 16.8. So 450 the interaction plot is showing how the RF and SGB models would extrapolate a combination of 451 variables not found in the training data.

452 In this case, the RF extrapolation, where importance is shared amongst multiple predictors, is 453 more sensible than the SGB model, where a single variable is driving the predictions. Since 454 TCC2001 is based off canopy cover predictions from a previous model, if that model had 455 erroneously classified some water as forest, the error would disproportionately affect the SGB 
456 model, as the SGB model for Utah is relying almost exclusively on that single variable (Figure $4574)$.

\section{$458 \quad 3.3$ Accuracy Measures}

\subsubsection{Histograms of plot frequency by Percent Canopy Cover}

460 We created histograms of plot frequency by percent canopy cover ( $1 \%$ classes) for the photo

461 plots in the independent test set. The height of the vertical bars represents the number of plots

462 found in each 1\% of TCC. These histograms were created for both the observed TCC and the

463 TCC predicted by the RF and SGB models.

464 For all four regions, the RF and SGB predictions for the test set had plot frequency histograms

465 similar to the observed data, and when the predictions failed to match the observed histograms,

466 both RF and SGB failed in similar ways (Figure 6).

467 The observed distribution of plots across low, medium and high canopy cover varies across the 468 four regions. The observed data in several regions had spikes at zero and or 100\% canopy cover.

469 Georgia and Oregon include plots across the full range of possible canopy cover, with spikes in

470 the number of plots at $0 \%$ and $100 \%$. Utah is similar, except that it has a higher proportion of

471 low canopy cover plots, and fewer plots with 100\% canopy cover. In Kansas, the majority of the

472 plots have very low canopy cover, with only a small number of plots at higher canopy cover.

473 Both models miss the canopy cover spike at zero in Oregon, and severely underestimate it in

474 Utah. Both models do capture the spike at zero canopy cover in Kansas, though they don't

475 predict quite as much low canopy cover as observed in the test data and tend to place the spike at 
476 very low canopy cover rather than at zero canopy cover. In Kansas, SGB places the spike at 2\%

477 cover and RF spreads the spike between zero and 3\% canopy cover.

478 Both models do an even worse job at capturing the observed spikes at 100\% canopy cover. In

479 Oregon the $100 \%$ canopy cover spike is reduced to a slight bump in frequency of plots with high

480 canopy cover. The models do better in Georgia, but the spike is still reduced slightly in

481 amplitude and shifted down from 100\% canopy cover and spread between 95-98\% cover for RF 482 and $95-97 \%$ cover for SGB.

\subsubsection{Error Statistics from the Independent Test Set}

484 There was very little difference in model performance between Random Forest and Stochastic 485 Gradient Boosting as measured by global accuracy metrics (Table 4). In all four study regions, 486 the independent test set MSE was identical to 3 decimal places, with the largest difference in 487 Kansas where RF gave an MSE of 0.0113 and SGB gave an MSE of 0.0117. Pearson's 488 correlation coefficient was identical to 2 decimal places, with Kansas again showing the largest 489 difference where RF had a correlation coefficient of 0.905 and SGB had 0.901. The Spearman's 490 correlation coefficient for RF was slightly worse than SGB in Kansas, but only by 0.01. The 491 largest difference in the predicted mean TCC was in Georgia, but they only differed by 0.004.

492 The largest difference in slope of the regression line was 0.03 in Oregon, and the largest 493 difference in intercept was 0.01 in Georgia, Oregon, and Utah.

494 Not only where the differences between RF and SGB models small, there also was no clear 495 pattern to which type of model performed best. In terms of MSE, RF was slightly better in 496 Kansas and Oregon, while SGB was slightly better in Georgia and Utah. In terms of Pearson's 497 correlation, RF was slightly better in Kansas, while SGB was slightly better in Georgia, Oregon 
498 and Utah. In terms of Spearman's correlation, RF was slightly better in Utah, while SGB was

499 slightly better in Kansas, Georgia and Oregon. Note that some of these differences were so small

500 as to be negligible. For example, in Utah the RF model had an MSE of 0.02976 and the SGB

501 model had 0.02977.

\section{$502 \quad 3.4$ Map Quality}

503 In Utah the final models were used to produce detailed TCC maps with predictions for every $50430 \mathrm{~m}$ pixel (Figure 7 and Figure 8). These figures illustrate the NAIP09 imagery (USDA 2009) 505 for portions of the Utah region, with the corresponding RF and SGB predicted TCC and the RF 506 uncertainty.

507 Histograms of number of pixels predicted by percent canopy cover for the Utah maps were 508 nearly identical for both RF and SGB.

509 The biggest difference between the RF and SGB maps was that the SGB model extrapolated 510 beyond the values of TCC found in the training data. The training data for UT had TCC ranging 511 from $0 \%$ to $100 \%$. The RF map predictions ranged from $-0.0001 \%$ to $97 \%$, approximately within

512 the range of the training data. In contrast, the SGB map predictions ranged from $-13 \%$ to $106 \%$.

513 In producing the final maps we treated predicted TCC values less than $0 \%$ as $0 \%$, and TCC

514 values greater than $100 \%$ as $100 \%$.

515 Overall, the maps for both models look similar, with RF picking up slightly more detail in the 516 regions of low crown cover. This highlights the need for potentially masking out non-forest areas

517 to eliminate spectrally dark anomalies in rangelands from being predicted erroneously as trees. 
518 The map of RF uncertainty highlighted small anomalous areas of high uncertainty. These are

519 localized areas where the predictions from individual trees varied widely, with some of the trees

520 in the Random Forest predicting low TCC, while other trees predicted high TCC. Two such

521 regions are examined in greater detail in Figure 7 and Figure 8. Figure 7 illustrates a lava bed, 522 while Figure 8 shows a wetland.

\section{4. Discussion}

524 A number of lessons were learned through the course of this study. First, we need to emphasize

525 the importance of having an independent tuning dataset, particularly for SGB models. We

526 increased our understanding of the selection of tuning parameters for RF and SGB models. We

527 also learned some interesting things about the effect of correlated predictor variables on variable

528 importance measures, and how these effects differ between RF and SGB.

\section{$529 \quad 4.1$ Importance of an independent tuning data set}

530 Tuning is a sometimes overlooked step in the model building process, both for traditional

531 parametric models, as well as for newer non-parametric models. Parametric models such as beta

532 regression depend on the choice of link function, preliminary removal of highly correlated

533 variables, and selection of final variables to include in both the model and, optionally, in the

534 precision equation. Non-parametric models do not require the elimination of non-significant

535 variables, but they do require optimizing model parameters, Stochastic Gradient Boosting more

536 so than Random Forest.

537 Model production is a two-step process: first, building potential models with a range of values of

538 model parameters; and second, selecting the combination of model parameters that gives the best 
539 performance as a final model. It is obvious that assessing a model over the data used to train the

540 model will not give a true estimate of performance on new independent data, as there will be no

541 way to distinguish over fitting from simple good performance. It is less obvious, but equally

542 hazardous to base a final model assessment on the data used for parameter selection. The

543 selected model could potential perform best on that particular data set, but not generalize to new

544 independent data. Therefore, particularly when working with complex models with large

545 numbers of parameters that need to be tuned, it is important to set aside both a tuning set, and

546 also a separate independent test set, to be used only for final model assessment.

547 If the final model can be built using only the training data then a tuning set is not necessary. If

548 decisions about the model are based on how well the model predicts over a dataset, then that

549 dataset can no longer be used to judge final model performance. With RF models, the default

550 parameters generally perform well (Liaw and Wiener 2002), therefore it is possible to accept the

551 default RF parameters, and use the entire dataset as training data and use Out-of-Bag for final

552 model evaluation. If a particular study does require tuning the RF parameters then Out-of-Bag

553 errors can be used for tuning, and an independent test set used for final model evaluation. With

554 SGB models, there is not an option for Out-of-Bag evaluation, and SGB models tend to be more

555 sensitive to model parameters, so having separate training, tuning, and test sets is more

556 important. With all types of models, the data that has been used to optimize the model cannot be

557 used for the final model evaluation.

\section{$558 \quad 4.2$ Tuning Process}

559 SGB models depend on a large number of parameters, and model performance can vary greatly

560 depending on the values chosen for these parameters. SGB models are also vulnerable to over 
561 fitting. If final model assessments are made on the same data as was used to select model

562 parameters, the true error may be considerably under estimated.

563 For SGB models, slower learning rates (smaller shrinkages) usually improve model performance,

564 but are computationally more expensive. There is also a point of diminishing returns where

565 dropping the shrinkage has less and less of an effect on model performance (Ridgeway 2007).

566 Shrinkage is inversely related to number of trees, so smaller values of shrinkage (slower learning

567 rates) require more trees. Authors agree on the inverse relationship, but opinions are divided on

568 whether this relationship scales evenly. For example dividing the learning rate by 2 might or

569 might not double the number of required trees (De'ath 2007, Ridgeway 2007, Elith et al. 2008).

570 For most of our regions, a shrinkage of 0.002 resulted in models with our desired number of trees

571 (3,000 to 5,000 trees), though Kansas required a lower (slower) shrinkage of 0.001 . Kansas is the

572 region with the highest proportion (53\%) of study sites with zero tree Canopy Cover.

573 It has been previously found that for presence-absence data, species with very high or very low

574 prevalence require lower values of shrinkage (Elith et al. 2008). When the majority of the data is

575 a single value, less information is available for each tree. In an extreme case, the random subset

576 selected for a tree could all have identical responses. A slower shrinkage allows a greater number

577 of trees to contribute to the final model before over fitting begins to occur. For continuous

578 response variables with high proportions of zero or $100 \%$ values a variation of this effect could

579 influence results in regions with large areas of non-forest, such as Kansas.

580 Very low prevalence could have a similar effect on bagging fraction. When a majority of the data 581 has a value of zero, larger bagging fractions help make certain that the points selected for each 
582 tree contain response values other than zero. Our results are consistant with this reasoning, as we

583 found that a higher bagging fraction (0.7) improved model predictions in Kansas.

584 Smaller bagging fractions introduce more stochastic uncertainty into the model, and therefore 585 can help counter-act over fitting (Friedman 2002). Oregon performed best with a lower bagging 586 fraction (0.2), which could suggest Oregon is somewhat more prone to overfitting . On the other

587 hand, it is also possible that the wide range of tree canopy cover present in Oregon meant that all 588 values of TCC were represented even in the smaller bagging fractions, and thus larger bagging 589 fractions offered less of an advantage in Oregon, as compared to regions such as Kansas where 590 the majority of the study sites had identical response values of zero and larger bagging fractions 591 may have been required to assure that all subsamples contained at least some non-zero response 592 data.

593 There are also potential draw backs to increased stochasticity that need to be kept in mind when 594 using models with low bagging fraction. (Note that faster shrinkages can also cause increased 595 stochasticity.) For example, increased stochasticity can result in higher between model 596 variability (Elith et al. 2008). Overall model performance may be similar between repeated 597 model runs, but predictions for individual locations may show high variation.

598 All of our regions performed best with a fairly high interaction depth. Shrinkage is inversely 599 related to interaction depth (Elith et al. 2008). A model with more complex trees will require 600 fewer total trees. On the other hand, complex trees make the model more vulnerable to over 601 fitting. Therefore it is recommended that as the tree complexity is increased, the learning rate 602 (shrinkage) is decreased so that the model fits slower and still requires a higher number of trees. 603 Elith et al. (2008) also notes that larger datasets can take better advantage of more complex trees. 
604 Our dataset was large enough that even our 50\% training data contained nearly 2000 data points 605 per region.

606 In contrast, RF models have only two parameters and are relatively insensitive to the choice of 607 these parameters (Liaw and Wiener 2002). The mtry parameter usually performs well at the 608 suggested default (one third the number of predictor variables for regression models). Our data

609 supports this. Two of our study regions did best with the suggested default mtry of 4, and in the 610 other two the slight improvement from the optimized mtry of 8 was only seen when the accuracy 611 measures were taken to three decimal places. In most cases RF is also less vulnerable to over 612 fitting than SGB, so the only limit on number of trees is computation time (Breiman 2001).

613 Therefore with RF model tuning is less imperative than with SGB. This, combined with the 614 possibility of using Out-of-Bag estimates of model quality, means that when faced with a small 615 dataset, RF can be used successfully without setting aside tuning or test data, simply building a 616 RF model on the full data set with the default parameters, and using Out-of-Bag estimates of 617 model performance.

\section{$618 \quad 4.3$ Comparing final model performance}

619 Our examination of relative variable importance elucidated the differences in how RF and SGB 620 make use of correlated predictor variables. Stochastic gradient boosting had a tendency to 621 concentrate variable importance in fewer variables, while Random Forest tended to spread 622 importance out amongst more variables. In Random Forest, each tree is independent, so if 623 predictor variables are highly correlated importance tends to be divided between the variables, 624 with one variable important to some of the trees, and the other variables important in other trees. 625 In SGB, each successive tree builds on the previous tree, so if variables are correlated, the first 
626 variable that is randomly selected is the most important, and even if other correlated variables are

627 chosen in later trees, there is less information they can contribute.

628 Both RF and SGB models had difficulty capturing spikes in crown cover at the extremes of the 629 distributions, either $0 \%$ or $100 \%$. Averaging inherent in both of these modeling techniques will 630 smooth the tails. This also indicates that global accuracy measures should be used with caution 631 and more information can be gained by examining the observed and predicted distributions.

632 Because this study is part of a larger project to update and improve the 2001 NLCD product, we 633 included tree canopy cover from the 2001 map as a predictor layer in our model. From the 634 variable importance graphs in Figure 4 it is clear that TCC2001 was an important variable for 635 both the RF and the SGB models. We did experiment with models that did not include TCC2001, 636 and surprisingly (given this predictors high importance in models built from the full set of 637 predictors) model performance only dropped slightly, with other predictor variables increasing in 638 importance when TCC2001 was not available. KS showed the strongest loss of model quality 639 from leaving out the TCC2001 predictor, and even there MSE from the RF model only increased 640 from 0.0113 to 0.0156 , and the MSE from the SGB model increased from 0.0117 to 0.0157 .

\section{$642 \quad 4.4$ Comparing Maps}

643 The RF uncertainty map for Utah is interesting, in that several small areas of unusually high 644 uncertainty were found. Figure 7 and Figure 8 examine two of these anomalies in detail. On 645 closer inspections both of these proved to be localized anomalies on the flat valley floors where 
646 both the RF and SGB models had predicted moderate to high crown cover. This was unusual as

647 in the Utah region flat, low elevation areas are most commonly sagebrush or other shrubland.

648 The RF uncertainty map is the standard deviation of the individual tree predictions from the final

649 RF model. High values of this standard deviation mean indicate a lack of agreement between the

650 trees. Each tree in a RF model is built from a different randomly selected subset of the predictor

651 variables, as well as a different bootstrap sample of the training locations. A high level of

652 uncertainty thus indicates that either particular predictors or particular training locations are

653 leading to high variation in the estimates for the response variable.

654 The black, irregularly shaped area in the northwest portion of Figure 7 is a lava bed. The bright 655 green circles to the east of the lava bed are agricultural. Both RF and SGB mistook the lava bed 656 for an area of moderate TCC (RF predicted slightly higher cover than SGB). The RF uncertainty 657 map shows that while the mean prediction from the 1500 trees in the model was for moderate 658 TCC, there was a very high level of uncertainty in these predictions, with some trees predicting 659 low TCC while other trees predicting much higher TCC.

660 The triangular area in the center of Figure 8 is a wetland - the Ke Bullock Waterfowl

661 Management Area. The rectangular green areas adjoining the wetland to the North and East are 662 agricultural. Both RF and SGB mistook the wetland for an area of moderate to high TCC. Again, 663 the RF uncertainty map shows that while the mean prediction from the in the RF model was for 664 moderate to high TCC, there was a very high level of uncertainty in these predictions.

665 In the final maps for UT (Figure 7 and Figure 8), it is also interesting to look at the predictions 666 and uncertainty for agricultural areas. In Figure 7, the RF uncertainty map shows some moderate 
667 uncertainty in the northern portion of the adjoining agricultural areas. While the LC and

668 TCC2001 predictors would indicate that these agricultural areas are non-forest, because of the

669 structure of RF models, some of the trees would be constructed from subsets of the predictors

670 containing neither LC nor TCC2001, leading to higher levels of between-tree RF uncertainty. In

671 contrast, the southern portion of the agricultural area in Figure 7 as well as the agricultural area

672 in Figure 8 were correctly identified as low TCC by both models, with low values of RF

673 uncertainty.

674 In addition to the lava bed, the uncertainty layer in Figure 7 also points out a rectangular

675 irregularity that can be traced back to the inconsistency of the national DEM layer, which

676 composites information from both lidar and large scale photography, depending on which is

677 available in different parts of the country. As a result, all the predictors that are derivatives from

678 the DEM (like slope, elev, aspect, CTI) witness these irregularities and hence influence the

679 uncertainty in the model. These irregularities may explain why the models ability to correctly

680 discern agriculture varied in different area of the map. It was the pattern in the uncertainty layer

681 here that first called attention to the more subtle patterning in the predictions themselves.

\section{5. Conclusions}

683 Random Forest and Stochastic Gradient Boosting are both powerful tree based modeling

684 techniques. We found that for our continuous response models the performance of both

685 techniques was remarkably similar on all four of our pilot regions, by all the accuracy measures

686 we examined. Therefore the choice of model type may come down to ease of use. 
687 In Random Forest, all the trees are independently built, while in Stochastic Gradient Boosting

688 each successive tree builds on the previous trees. Both provide importance measures for the

689 predictor variables. Random Forest is more user friendly than Stochastic Gradient Boosting, as it

690 has fewer parameters to be set by the user, and is less sensitive to tuning of these parameters.

691 Random Forest is also less prone to over fitting than Stochastic Gradient Boosting. In Random

692 Forests, using additional trees increases the time and computations, but does not usually lead to

693 loss of model quality.

694 Random Forest has an out-of-bag option for model evaluation without the necessity of setting

695 aside an independent test set. Combine this with Random Forest's lack of sensitivity to model

696 parameters and it is possible to build and evaluate a model from the full dataset, without setting

697 aside tuning or test data. This can be an important advantage over Stochastic Gradient Boosting,

698 particularly for small datasets.

699 In contrast to Random Forest, Stochastic Gradient Boosting has many parameters needing

700 tuning, and these parameters have stronger effects on model quality. Also, over fitting is much

701 more likely with Stochastic Gradient Boosting, and the number of trees that will lead to over

702 fitting changes with the values of the other parameters.

703 Additionally, Random Forest offers the possibility of a map of the stochastic uncertainty

704 remaining in the final model, which we found valuable for identifying possibly anomalous areas

705 in the final map.

706 As a result of these and other analyses, random forests models are currently being used by the

707 US Forest Service Remote Sensing Applications Center (http://www.fs.fed.us/eng/rsac/) to 
708 produce the 2011 NLCD percent tree canopy cover dataset for the conterminous United

709 States. This dataset is publically available at the NLCD website (http://www.mrlc.gov/). Percent

710 canopy cover datasets for coastal Alaska will be finished Spring of 2015, and it is planned to

711 have interior Alaska, Hawaii, Virgin Islands, and Puerto Rico completed near the end of 2015.

\section{6. Acknowledgements}

713 The authors would like to acknowledge the US Forest Service, Forest Inventory and Analysis

714 program for support. Thanks also go out to staff at the US Forest Service Remote Sensing

715 Applications Center.

\section{7. References}

717 Baccini, A., Laporte, N., Goetz, S. J., Sun, M., \& Dong, H. 2008. A first map of tropical Africa's

718 above-ground biomass derived from satellite imagery. Environmental Research Letters, 3(4), 719045011.

720 Bailey, R. G. 1995. Description of the Ecoregions of the United States, U.S.D.A. Forest Service

721 Misc. Pub. 1391, U.S. D. A. Forest Service, Washington.

722 Baker, C., Lawrence, R., Montagne, C., and Patten, D.. 2006. Mapping wetlands and riparian

723 areas using landsat etm+ imagery and decision-tree-based models. Wetlands 26 (2): 465-474

724 Bechtold, W.A., and Patterson, P.L. (editors), 2005. The Enhanced Forest Inventory and 725 Analysis Program - National Sampling Design and Estimation Procedures, USDA Forest 
726 Service, General Technical Report SRS-80, Southern Research Station, Asheville, North

727 Carolina, $85 \mathrm{p}$.

728 Breiman, L., Friedman, R. A., Olshen, R. A., and Stone, C. G. 1984. Classification and 729 Regression Trees. Wadsworth, 1984.

730 Breiman, L. 2001. Random forests. Mach. Learn. 45: 5-32.

731 Chan, J. C. W., and Paelinckx, D. 2008. Evaluation of random forest and adaboost tree-based

732 ensemble classification and spectral band selection for ecotype mapping using airborne

733 hyperspectral imagery Remote Sensing of Environment, 112(6) 2999-3011.

734 Chirici, G., Scotti, R., Montaghi, A., Barbati, A., Cartisano, R., Lopez, G., Marchetti, M.,

735 McRoberts, R.E., Olsson, H., \& Corona, P. 2013. Stochastic gradient boosting classification trees

736 for forest fuel types mapping through airborne laser scanning and IRS LISS-III imagery.

737 International Journal of Applied Earth Observation and Geoinformation, 25: 87-97.

738 Cochran, W.G., 1977. Sampling Techniques, Third edition, John Wiley \& Sons, New York, 428 739 p.

740 Coulston, J. W., Moisen, G. G., Wilson, B. T., Finco, M. V., Cohen, W. B., and Brewer, C. K. 741 2012. Modeling percent tree canopy cover: A pilot study. Photogrammetric Engineering and 742 Remote Sensing 78(7): 715-727.

743 De'ath, G., and Fabricius, K. E. 2000. Classification and regression trees: a powerful yet simple 744 technique for ecological data analysis. Ecology, 81(11): 3178-3192. 
745 De'ath, G.. 2007. Boosted trees for ecological modeling and prediction. Ecology, 88(1), 243-51.0

746 Elith, J., Leathwick, J. R., and Hastie, T.. 2008. A working guide to boosted regression trees.

747 Journal of Animal Ecology, 77: 802-13.

748 Evans, J., and Cushman, S.. 2009. Gradient modeling of conifer species using random forests.

749 Landscape Ecology, 24: 673-683.

750 Filippi, A. M., Güneralp, İ., and Randall, J. 2014. Hyperspectral remote sensing of aboveground

751 biomass on a river meander bend using multivariate adaptive regression splines and stochastic

752 gradient boosting. Remote Sensing Letters 5(5): 432-441, doi:10.1080/2150704X.2014.915070.

753 Freeman, E. and Frescino, T.. 2009. ModelMap: An R Package for Modeling and Map

754 production using Random Forest and Stochastic Gradient Boosting. USDA Forest Service,

755 Rocky Mountain Research Station, 507 25th street, Ogden, UT, USA.

756 Frescino, T. S., and Moisen, G. G. 2012. Comparing alternative tree canopy cover estimates

757 derived from digital aerial photography and field-based assessments. In Monitoring Across

758 Borders: 2010 Joint Meeting of the Forest Inventory and Analysis (FIA) Symposium and the

759 Southern Mensurationists. Edited by W. McWilliams and F. A. Roesch, e-Gen. Tech. Rep. SRS-

760 157. Asheville, NC: U.S. Department of Agriculture, Forest Service, Southern Research Station.

761 pp 237-244

762 Friedman, J., Hastie, T., and Tibshirani, R. 2000. Additive logistic regression: a statistical view

763 of boosting. Ann. Statist. 28(2): 337-407. 
764 Friedman, J. H. 2001. Greedy function approximation: a gradient boosting machine. Ann. Stat. 765 29(5): 1189-1232.

766 Friedman, J. H. 2002. Stochastic Gradient Boosting. Computational Statistics and Data Analysis, $767 \quad 28: 367-78$.

768 Gislason, P. O., Benediktsson, J. A., and Sveinsson, J. R. 2006. Random Forests for land cover 769 classification. Pattern Recognition Letters, 27(4): 294-300.

770 Güneralp, İ., Filippi, A. M., and Randall, J. 2014. Estimation of floodplain aboveground biomass

771 using multispectral remote sensing and nonparametric modeling. International Journal of

772 Applied Earth Observation and Geoinformation 33: 119-126,

773 http://dx.doi.org/10.1016/j.jag.2014.05.004.

774 Hastie, T., Tibshirani, R., and Friedman, J. 2009. Model Assessment and Selection. In: The

775 Elements of Statistical Learning, Springer Series in Statistics. New York: Springer. pp. 219-259.

776 Homer, C. Huang, C., Yang, L., Wylie, B., and Coan, M. 2004. Development of a 2001 National

777 Landcover Database for the United States. Photogrammetric Engineering and Remote Sensing,

778 Vol. 70, No. 7, July 2004, pp. 829-840.

779 Hauke, J., \& Kossowski, T. 2011. Comparison of values of Pearson's and Spearman's correlation 780 coefficients on the same sets of data. Quaestiones geographicae, 30(2), 87-93.

781 Homer, C., Dewitz, J., Fry, J., Coan, M., Hossain, N., Larson, C., Herold, N., McKerrow, A., 782 VanDriel, J.N., and Wickham, J.. 2007. Completion of the 2001 National Land Cover Database 
783 for the conterminous United States, Photogrammetric Engineering \& Remote Sensing, 73(4):

$784 \quad 337-341$.

785 Jackson, T. A., Moisen, G., Patterson, P. L., and Tipton, J. 2012. Repeatability in photo-

786 interpretation of tree canopy cover and its effect on predictive mapping. In Monitoring Across

787 Borders: 2010 Joint Meeting of the Forest Inventory and Analysis (FIA) Symposium and the

788 Southern Mensurationists. Edited by W. McWilliams and F. A. Roesch, e-Gen. Tech. Rep. SRS-

789 157. Asheville, NC: U.S. Department of Agriculture, Forest Service, Southern Research Station.

790 pp. $189-192$

791 Jennings, S. B., Brown, N. D., and Sheil, D. 1999. Assessing forest canopies and understory

792 illumination: canopy closure, canopy cover and other measures, Forestry 72(1): 59-73.

793 Kellndorfer, J. M., Walker, W., LaPoint, E., Hoppus, M., and Westfall, J. 2006. Modeling

794 Height, Biomass, and Carbon in US Forests from FIA, SRTM, and Ancillary National Scale Data

795 Sets, Proceedings of the IEEE International Symposium on Geoscience and Remote Sensing

796 (IGARSS), July 31- August 4 2006, Denver, CO, pp. 3591-3594.

797 Lawrence, R., Bunn, A., Powell, S., and Zambon, M. 2004.Classification of remotely sensed

798 imagery using stochastic gradient boosting as a refinement of classification tree analysis. Remote

799 Sensing of Environment 9(3): 331-336.

800 Lawrence, R. L., Wood, S. D., and Sheley, R. L. 2006. Mapping invasive plants using

801 hyperspectral imagery and Breiman Cutler classifications (Random Forest). Remote Sensing of

802 Environment 100: 356 - 362. 
803 Leathwick, J. R., Elith, J., Francis, M. P., Hastie, T., and Taylor, P. 2006. Variation in demersal

804 fish species richness in the oceans surrounding New Zealand: an analysis using boosted

805 regression trees. Mar Ecol Prog Ser, 321: 267-281

806 Liaw, A., and Wiener, M.. 2002. Classification and regression by random forest. R News2, 18

807 22. Available from: http://CRAN.R-project.org/doc/Rnews/.

808 McRoberts, R.E., Holden, G.R., Nelson, M.D., Liknes, G.C., and Gormanson, D.D., 2006. Using

809 satellite imagery as ancillary data for increasing the precision of estimates for the Forest

810 Inventory and Analysis program of the USDA Forest Service, Canadian Journal of Forest

811 Research, 36:2968-2980.

812 Moisen G. G. and Frescino, T. S. 2002. Comparing five modelling techniques for predicting

813 forest characteristics. Ecological Modeling 157: 209 - 225.

814 Moisen, G. G., Freeman, E. A., Blackard, J.A., Frescino, T. S., Zimmermann, N. E., and

815 Edwards Jr, T. C. 2006. Predicting tree species presence and basal area in Utah: A comparison of

816 stochastic gradient boosting, generalized additive models, and tree-based methods. Ecological

817 Modelling 199: 176-187.

818 Moisen, G. G. 2008. Classification and regression trees. In Encyclopedia of Ecology, volume 1.

819 Edited by S. E. Jørgensen and B. D. Fath, Elsevier. pp. 582-588.

820 Moisen, G. G., Coulston, J. W., Wilson, B. T., Cohen, W. B., and Finco, M. V. 2012. Choosing

821 appropriate subpopulations for modeling tree canopy cover nationwide. In Monitoring Across

822 Borders: 2010 Joint Meeting of the Forest Inventory and Analysis (FIA) Symposium and the 
823 Southern Mensurationists. Edited by W. McWilliams and F. A. Roesch, e-Gen. Tech. Rep. SRS-

824 157. Asheville, NC: U.S. Department of Agriculture, Forest Service, Southern Research Station. 825 pp. 195-200.

826 Moore I. D., Grayson, R. B., and Ladson, A. R. 1991. Digital terrain modelling: review of

827 hydrological, geomorphological, and biological applications. Hydrological Processes, 5: 3-30.

828 Nowak, D. J., Crane, D. E., and Stevens, J. C. 2006. Air pollution removal by urban trees and 829 shrubs in the United States, Urban Forestry and Urban Greening, 4(3-4): 115-123.

830 Pittman, S. J., Costa, B. M., and Battista, T. A. 2009. Using Lidar Bathymetry and Boosted 831 Regression Trees to Predict the Diversity and Abundance of Fish and Corals. Journal of Coastal 832 Research: Special Issue 53: $27-38$.

833 Powell, S. L., Cohen, W. B., Healey, S. P., Kennedy, R. E., Moisen, G. G., Pierce, K. B., and

834 Ohmann, J. L. 2010. Quantification of live aboveground forest biomass dynamics with Landsat 835 time-series and field inventory data: A comparison of empirical modeling approaches, Remote 836 Sensing of Environment, 114(5): 1053-1068,

837 Prasad, A. M., Iverson, L. R., and Liaw, A.. 2006. Newer classification and regression tree 838 techniques: bagging and random forests for ecological prediction. Ecosystems, 9: 181-99.

839 R Development Core Team. 2008. R: A Language and Environment for Statistical Computing. R 840 Foundation for Statistical Computing, Vienna, Austria. URL http://www.R-project.org. ISBN 3$841900051-07-0$.

842 Ridgeway, G. 1999. The state of boosting. Computing Science and Statistics, 31, 172-181. 
843 Ridgeway, G. 2007. Generalized boosted models: a guide to the gbm package. Available at

844 http://cran.r-project.org/web/packages/gbm/vignettes/gbm.pdf

845 Greg Ridgeway with contributions from others (2013). gbm: Generalized Boosted Regression

846 Models. R package version 2.1. http://CRAN.R-project.org/packages/gbm

847 Rollins, M.G., and Frame, C.K. (Editors). 2006. The LANDFIRE Prototype Project: nationally

848 consistent and locally relevant geospatial data for wildland fire management, USDA Forest

849 Service, General Technical Report RMRS-GTR-175, Rocky Mountain Research Station, Fort

850 Collins, CO, 416 p.

851 Sankaran, M., Ratnam, J., and Hanan, N.. 2008. Woody cover in African savannas: the role of

852 resources, fire and herbivory. Global Ecology and Biogeography, 17: 236-245.

853 Tipton, J., Moisen, G., Patterson, P., Jackson, T. A., and Coulston, J. 2012. Sampling intensity

854 and normalizations: Exploring cost-driving factors in nationwide mapping of tree canopy cover.

855 In: Monitoring Across Borders: 2010 Joint Meeting of the Forest Inventory and Analysis (FIA)

856 Symposium and the Southern Mensurationists. Edited by W. McWilliams and F. A. Roesch, e-

857 Gen. Tech. Rep. SRS-157. Asheville, NC: U.S. Department of Agriculture, Forest Service,

858 Southern Research Station. pp. 201-208.

859 Toney, C., Liknes, G., Lister, A., and Meneguzzo, D. 2012. Assessing alternative measures of

860 tree canopy cover: Photo-interpreted NAIP and ground-based estimates. In: Monitoring Across

861 Borders: 2010 Joint Meeting of the Forest Inventory and Analysis (FIA) Symposium and the

862 Southern Mensurationists. Edited by W. McWilliams and F. A. Roesch, e-Gen. Tech. Rep. SRS- 
863 157. Asheville, NC: U.S. Department of Agriculture, Forest Service, Southern Research Station. 864 pp. 209-215.

865 U.S. Department of Agriculture, 2009. National Agriculture Imagery Program, URL:

866 http://www.apfo.usda.gov/FSA/apfoapp?area=home\&subject=prog\&topic=nai, USDA Farm

867 Service Agency, Aerial Photography Field Office, Salt Lake City, Utah (last date Accessed: 29

868 March 2012).

869 Webb, B.W., and Crisp, D. T. 2006. Afforestation and stream temperature in a temperate

870 maritime environment, Hydrological Processes, 20(1):51-66.

871 White, D., Kimerling, J., and Overton, S.W., 1992. Cartographic and geometric components of a

872 global sampling design for environmental monitoring, Cartographic and Geographic Information

873 Systems, 19(1):5-22. 
RF AND SGB MODELS FOR TREE CANOPY COVER

PAGE 44

\section{Tables}

876 Table 1 Percent of photo plots per NLCD land cover class for the four study regions.

\begin{tabular}{clcccc}
\hline & & \multicolumn{4}{c}{ Study Regions } \\
\cline { 3 - 5 } Code & Land Cover Class & GA & KS & OR & UT \\
\hline 11 & Open Water & $2 \%$ & $2 \%$ & $1 \%$ & $0 \%$ \\
12 & Perennial Ice/Snow & $0 \%$ & $0 \%$ & $0 \%$ & $0 \%$ \\
21 & Developed Open Space & $11 \%$ & $4 \%$ & $2 \%$ & $1 \%$ \\
22 & Developed Low Intensity & $6 \%$ & $2 \%$ & $1 \%$ & $0 \%$ \\
23 & Developed Medium Intensity & $2 \%$ & $0 \%$ & $0 \%$ & $0 \%$ \\
24 & Developed High Intensity & $1 \%$ & $0 \%$ & $0 \%$ & $0 \%$ \\
31 & Barren Land & $1 \%$ & $0 \%$ & $1 \%$ & $4 \%$ \\
41 & Deciduous Forest & $39 \%$ & $8 \%$ & $0 \%$ & $4 \%$ \\
42 & Evergreen Forest & $19 \%$ & $0 \%$ & $45 \%$ & $35 \%$ \\
43 & Mixed Forest & $1 \%$ & $0 \%$ & $2 \%$ & $2 \%$ \\
52 & Shrub/Scrub & $1 \%$ & $0 \%$ & $36 \%$ & $45 \%$ \\
71 & Grassland/Herbaceous & $3 \%$ & $41 \%$ & $2 \%$ & $3 \%$ \\
81 & Pasture/Hay & $12 \%$ & $17 \%$ & $5 \%$ & $3 \%$ \\
82 & Cultivated Crops & $0 \%$ & $24 \%$ & $4 \%$ & $1 \%$ \\
90 & Woody Wetlands & $2 \%$ & $1 \%$ & $0 \%$ & $0 \%$ \\
95 & Emergent Herbaceous Wetlands & $0 \%$ & $0 \%$ & $1 \%$ & $0 \%$ \\
\hline
\end{tabular}


878 Table 2 Mean percent Tree Canopy Cover (TCC) and the standard error of the mean (s.e.(TCC)) for each study area for 879 NLCD2001 forest land cover urban land cover, and across all land cover classes based on photo interpretation.

880 Reproduced with permission from Coulston, et al. (2012).

\begin{tabular}{lrcc}
\hline \multirow{2}{*}{ Study Region } & \multicolumn{2}{c}{ Sample Based } \\
\cline { 3 - 4 } GA & Forest & 84.1 & 0.45 \\
& Urban & 41.1 & 0.94 \\
& All & 66.0 & 0.53 \\
\hline KS & Forest & 71.0 & 1.57 \\
& Urban & 14.5 & 1.28 \\
& All & 12.8 & 0.40 \\
\hline OR & Forest & 66.5 & 0.60 \\
& Urban & 26.4 & 2.33 \\
& All & 41.6 & 0.51 \\
\hline UT & Forest & 52.0 & 0.68 \\
& Urban & 9.1 & 1.68 \\
& All & 27.4 & 0.47 \\
\hline
\end{tabular}

881 
883 Table 3 Predictor variable for models. For predictor variables originated from 30 m products, assignment to each 90 m 884 plot was accomplished by taking a focal mean or focal majority over a $3 \times 3$ window for continuous variables, and focal 885 majority for the categorical variables. See Coulston et al. (2012) for more details on the predictor variables.

\begin{aligned} & \hline Predictors \multicolumn{1}{c}{ Description } \\ & \hline NORTHNESS Northness - cos(aspect) \\ & EASTNESS Eastness - sin(aspect) \\ & SLOPE slope \\ & CTI topographic positional index \\ & ELEV elevation \\ & ECOREG Bailey's ecoregions \\ & LC land cover class from 2001 NLCD \\ & TCC2001 tree canopy cover from 2001 NLCD \\ & BAND1 Landsat-5 band 1 - Blue \\ & BAND2 Landsat-5 band 2 - Green \\ & BAND3 Landsat-5 band 3 - Red \\ & BAND4 Landsat-5 band 4 - Near IR \\ & BAND5 Landsat-5 band 5 - Short-wave IR \\ & BAND7 Landsat-5 band 7 - Short-wave IR \\ & \hline\end{aligned}


888

889

890

Table 4 Independent test set error statistics from final models: mean square error, Pearson's correlation, and Spearman's correlation. Pearson's correlation is a measure of linear correlation, while Spearman's correlation measures monotonic non-linear correlation.

\begin{tabular}{lrcc}
\hline & & \multicolumn{2}{c}{ Model } \\
\cline { 3 - 4 } \multicolumn{2}{c}{ Study Region } & SGB & RF \\
\hline GA & MSE & 0.0185 & 0.0188 \\
& Pearson's & 0.919 & 0.917 \\
& Spearman's & 0.882 & 0.881 \\
\hline KS & MSE & 0.0117 & 0.0113 \\
& Pearson's & 0.901 & 0.905 \\
& Spearman's & 0.673 & 0.662 \\
\hline OR & MSE & 0.0246 & 0.0246 \\
& Pearson's & 0.896 & 0.896 \\
& Spearman's & 0.850 & 0.880 \\
\hline UT & MSE & 0.0298 & 0.0298 \\
& Pearson's & 0.833 & 0.833 \\
& Spearman's & 0.850 & 0.850 \\
\hline
\end{tabular}

891

892 
894 Table 5 SGB model parameters as used by various authors.

\begin{tabular}{|l|l|l|l|}
\hline \multicolumn{1}{|c|}{ Friedman (2002) } & \multicolumn{1}{|c|}{ Elith et al. (2008) } & \multicolumn{1}{c|}{ Ridgeway (2007) } & \multicolumn{1}{c|}{ gbm package } \\
\hline \multicolumn{1}{|c|}{$\begin{array}{c}\text { stochastic gradient } \\
\text { boosting }\end{array}$} & boosted regression trees & generalized boosted models & $\begin{array}{c}\text { generalized boosted } \\
\text { models }\end{array}$ \\
\hline $\begin{array}{l}\text { error distribution } \\
\text { M - iterations }\end{array}$ & response type & distribution & distribution \\
$\begin{array}{l}\text { L - tree size/ number of } \\
\text { terminal nodes }\end{array}$ & $\begin{array}{l}\text { nt - number of trees } \\
\text { number of nodes }\end{array}$ & $\mathrm{T}$ - number of iterations & n.trees \\
$v$ - shrinkage & lr - learning rate & $\lambda$ - learning rate & interaction.depth \\
f-sampling fraction & bag fraction & $\mathrm{p}$ - subsampling/bagging rate & bag.fraction \\
\hline
\end{tabular}




\section{Figure Captions}

897 Figure 1. Tuning RF - effect of mtry and ntree on RF models for Georgia and Kansas. Each line

898 indicates one model as the number of trees is increased. In each region twenty models were fit

899 for each of three values of mtry (line shade indicates mtry). The error is plotted as a function of

900 the number of trees in each model. As ntree increases the stochastic uncertainty of the models

901 decreases and the lines for a given value of mtry converge. Good models have a low MSE and

902 high Pearson's and Spearman's correlation. MSE is shown both on the tuning data and on the

903 training data. In Georgia, mtry of 2 and 4 had lowest MSE, with mtry if 2 having highest Pearson

904 correlation and mtry of 4 having highest Spearman correlation. In Kansas mtry of 8 was best by

905 all three measures. These RF models do not show evidence of overfitting with increasing

906 numbers of trees, as both the tuning and the training MSE initially decrease, and then remain

907 stable as trees are added to the models.

908 Figure 2. Tuning SGB - effect of interaction depth (tree complexity) and bagging fraction on

909 SGB models of Georgia. Points represent best number of trees for each combination of

910 parameters. Final model was constructed with shrinkage $=0.002$, bagging fraction $=0.40$ and

911 interaction depth=10.

912 Figure 2 Tuning SGB - Effects of shrinkage (learning rate) and bagging fraction on tuning set

913 error rates in Georgia and Kansas. All other model parameters optimized for each region. Notice

914 that the same shrinkage rate requires more trees to reach the best model performance in Georgia

915 than in Kansas. Our goal of 3000-5000 trees required a shrinkage rate of 0.002 in Georgia and

9160.001 in Kansas. In Georgia, the best bagging fraction is between 0.3 and 0.5 (we used 0.4 in the 
917 final model). In Kansas, 0.7 and 0.9 tie for best bagging fraction (we used 0.7 is the final model).

918 Notice also that the error line for models with low bagging fractions $(0.1$ and 0.3$)$ jitters slightly

919 due to increased stochastic uncertainty. The SGB models show evidence of overfitting with

920 increasing number of trees, particularly with higher values of shrinkage. The tuning error

921 initially decreases as trees are added, but then as more trees are added the tuning error begins to

922 rise again. This illustrates why with SGB models it is essential to optimize the number of trees

923 used for the final model.

924 Figure 4. Variable importance for the RF and SGB models of the 4 pilot regions. RF on the left

925 in gray, and SGB on the right in black. RF importance measured by the percent increase in MSE

926 with random permutation of each variable. SGB importance measured by the decrease in squared

927 error attributed to each variable in the gradient of the loss function. Variable importance of each

928 model scaled to sum to 1.

929 Figure 5. Interaction plots for elevation (ELEVATION) and land cover class (LC) for final Utah

930 models. These figures show the effect of changes in two predictor variables on predicted TCC,

931 with all other variables held at their mean (or majority). To see an example of interaction, look at

932 the RF model, and the effect of elevation in land cover classes 41 (Deciduous Forest) and 42

933 (Evergreen Forest). In class 41, TCC is moderate at the lowest elevations in the UT region,

934 increases at mid elevations, and drops slightly to moderate levels at high elevations. In class 42 ,

935 TCC starts slightly higher than class 41 at low elevations, again rises at mid elevations, but then

936 drops to near zero at high elevations. Also notice the differences between the RF model and the

937 SGB model. Even though overall accuracy of the two models is similar, the predicted TCC in

938 Land Cover Class 11 (open water) is very different. 
939 Figure 6. Histograms illustrating number of photo plots in the independent test sets by percent

940 tree canopy cover (1\% classes). The observed plot distribution varies across the four regions.

941 Georgia and Oregon include plots across the full range of possible canopy cover, with spikes in

942 the number of plots at $0 \%$ and $100 \%$. Utah is similar, except that it has a higher proportion of

943 low canopy cover plots, and fewer plots with 100\% canopy cover. In Kansas, the majority of the

944 plots have very low canopy cover, with only a small number of plots at higher canopy covers.

945 Both RF and SGB models had difficulty capturing the observed spikes in number of plots at $0 \%$ 946 and $100 \%$.

947 Figure 7. Detail map of northwest portion of the Utah region, showing NAIP09 imagery, The RF 948 Uncertainty map, and Random Forest and Stochastic Gradient Boosting predictions for Tree

949 Canopy Cover. The dark patch in the upper left of the photo is a lava bed. Both RF and SGB 950 mistook it for an area of moderate TCC (RF predicted slightly higher cover than SGB). The RF 951 uncertainty map shows that while the mean prediction from the 1500 trees in the model was for 952 moderate TCC, there was a very high level of uncertainty in these predictions, with some trees 953 predicting low TCC while other trees predicting much higher TCC.

954 Figure 8. Detail map of eastern portion of the Utah region, showing NAIP09 imagery, The RF 955 Uncertainty map, and Random Forest and Stochastic Gradient Boosting predictions for Tree 956 Canopy Cover. The dark triangular patch in the middle of the photo is a wetland. Both RF and 957 SGB mistook it for an area of moderate to high TCC. The RF uncertainty map shows that while 958 the mean prediction from the 1500 trees in the model was for moderate to high TCC, there was a 959 very high level of uncertainty in these predictions, with some trees predicting low TCC while 
960 other trees predicting much higher TCC. The adjoining agricultural area was correctly identified

961 as low TCC by both models, most likely due to the TCC2001 and the LC predictor layers. 

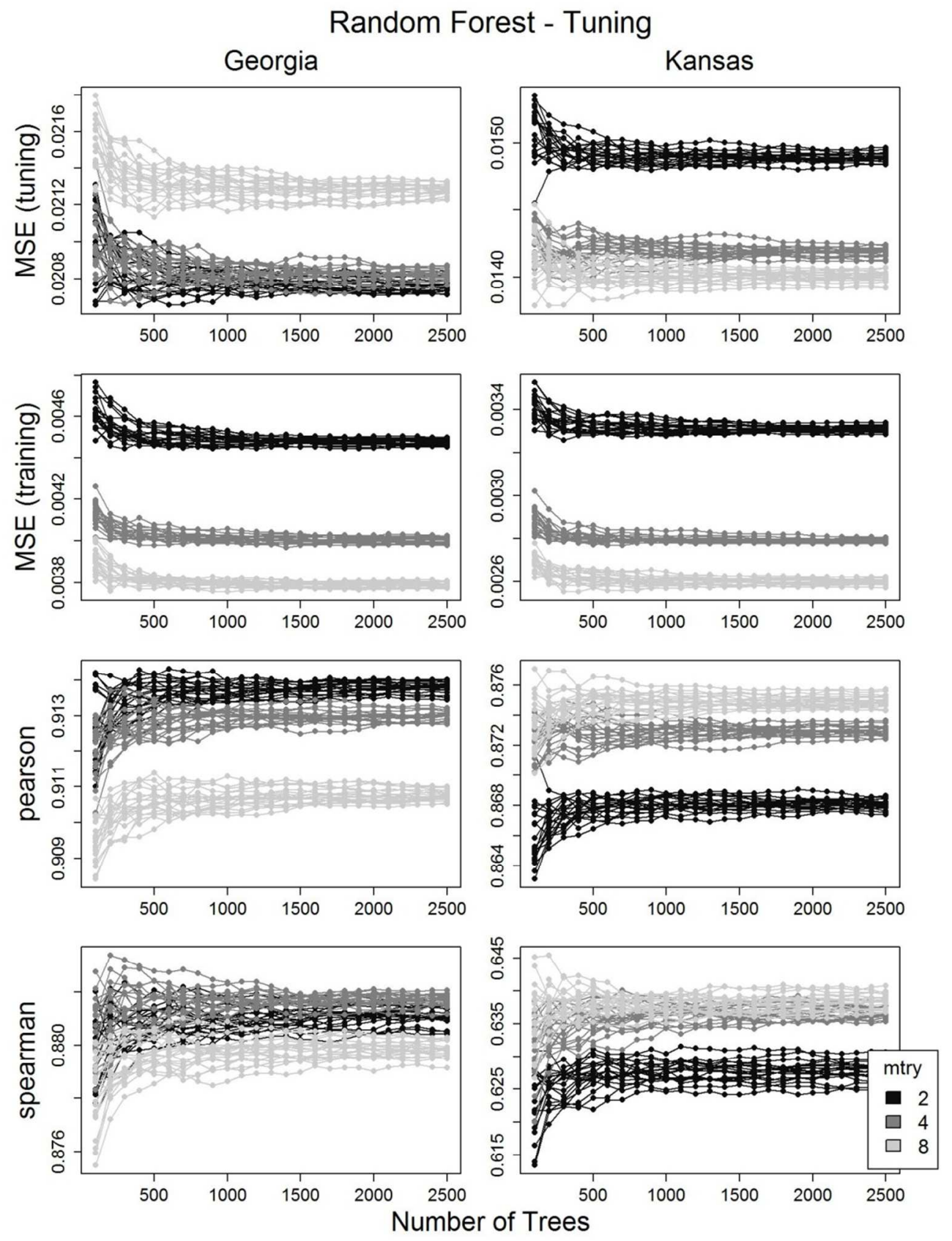

Figure 1 Tuning RF - effect of mtry and ntree on RF models for Georgia and Kansas. Each line indicates one model as the number of trees is increased. In each region twenty models were fit for each of three values of mtry (line shade indicates mtry). The error is plotted as a function of the number of trees in each model. As ntree increases the stochastic uncertainty of the models decreases and the lines for a given value of mtry converge. Good models have a low MSE and high Pearson's and Spearman's correlation. MSE is shown both on the tuning data and on the training data. In Georgia, mtry of 2 and 4 had lowest MSE, with mtry if 2 having highest Pearson correlation and mtry of 4 having highest Spearman correlation. In Kansas mtry of 8 was best by all three measures. These RF models do not show evidence of overfitting with increasing numbers of trees, as both the tuning and the training MSE initially decrease, and then remain stable as trees are added to the models. 

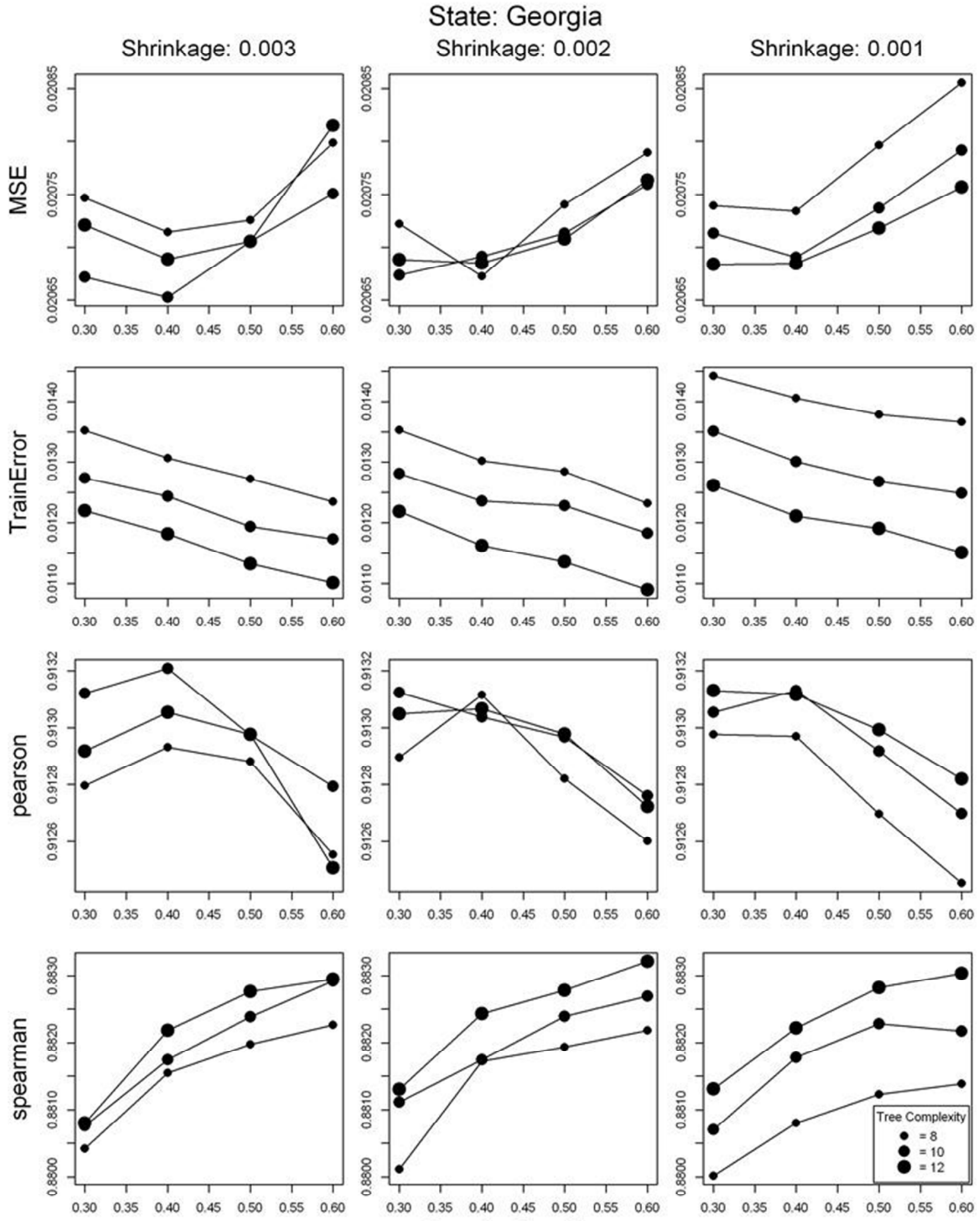

Bagging Fraction

Figure 3 Tuning SGB - effect of interaction depth (tree complexity) and bagging fraction on SGB models of Georgia. Points represent best number of trees for each combination of parameters. Final model was constructed with shrinkage $=0.002$, bagging fraction $=0.40$ and interaction depth $=10$. 


\section{Effect of Shrinkage on Error Rate}
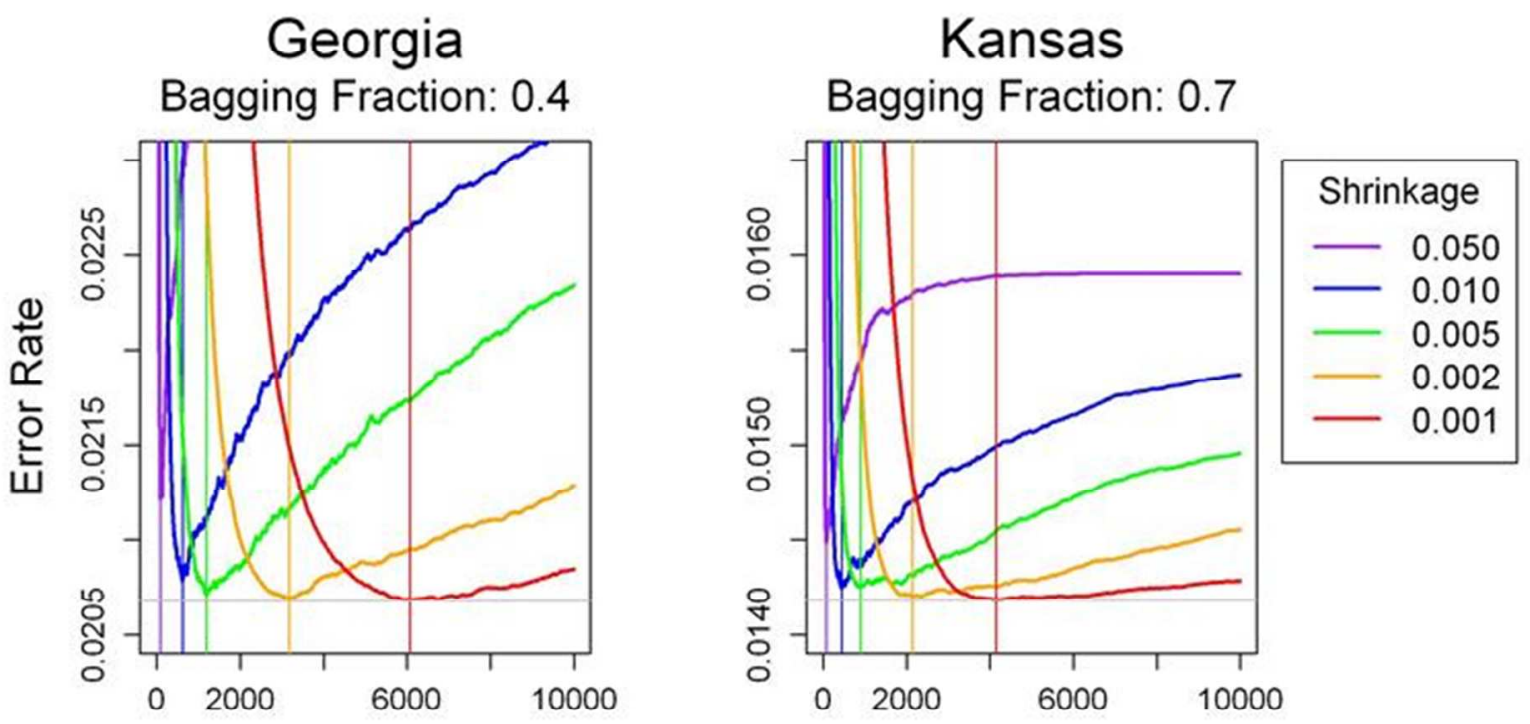

\section{Effect of Bagging Fraction on Error Rate \\ Georgia \\ Kansas}

Shrinkage: 0.002

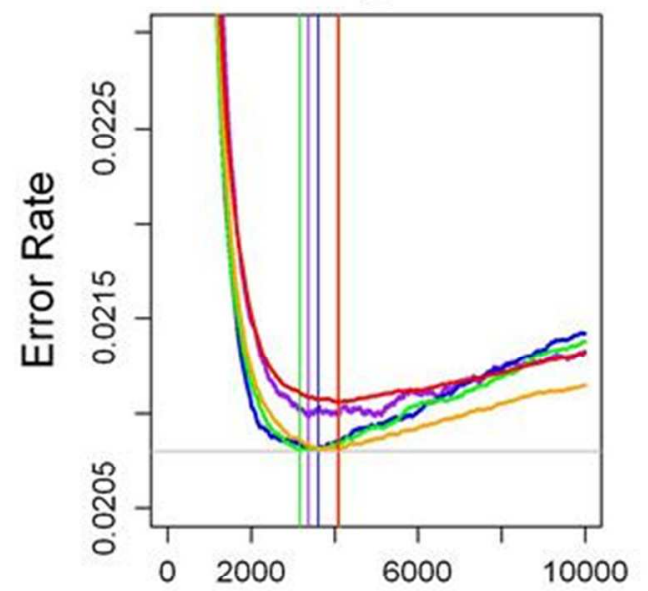

Shrinkage: 0.001

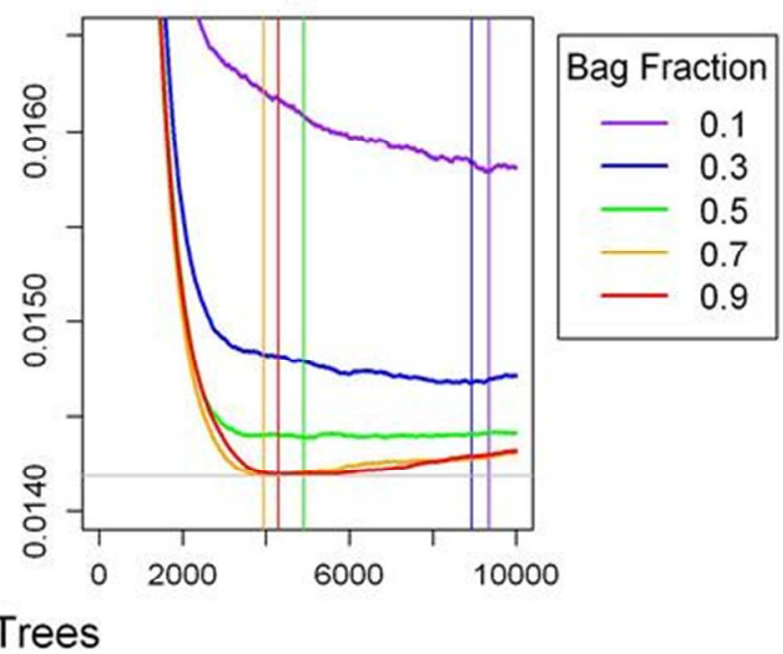

976
Figure 3 Tuning SGB - Effects of shrinkage (learning rate) and bagging fraction on tuning set error rates in Georgia and Kansas. All other model parameters optimized for each region. Notice that the same shrinkage rate requires more trees to reach the best model performance in Georgia than in Kansas. Our goal of 3000-5000 trees required a shrinkage rate of 0.002 in Georgia and 0.001 in Kansas. In Georgia, the best bagging fraction is between 0.3 and 0.5 (we used 0.4 in the final model). In Kansas, 0.7 and 0.9 tie for best bagging fraction (we used 0.7 is the final model). Notice also that the error line for models with low bagging fractions $(0.1$ and 0.3$)$ jitters slightly due to increased stochastic uncertainty. The SGB models show evidence of overfitting with increasing number of trees, particularly with higher values of shrinkage. The tuning error initially decreases as trees are added, but then as more trees are added the tuning error begins to rise again. This illustrates why with SGB models it is essential to optimize the number of trees used for the final model. 


\section{Variable Importance}

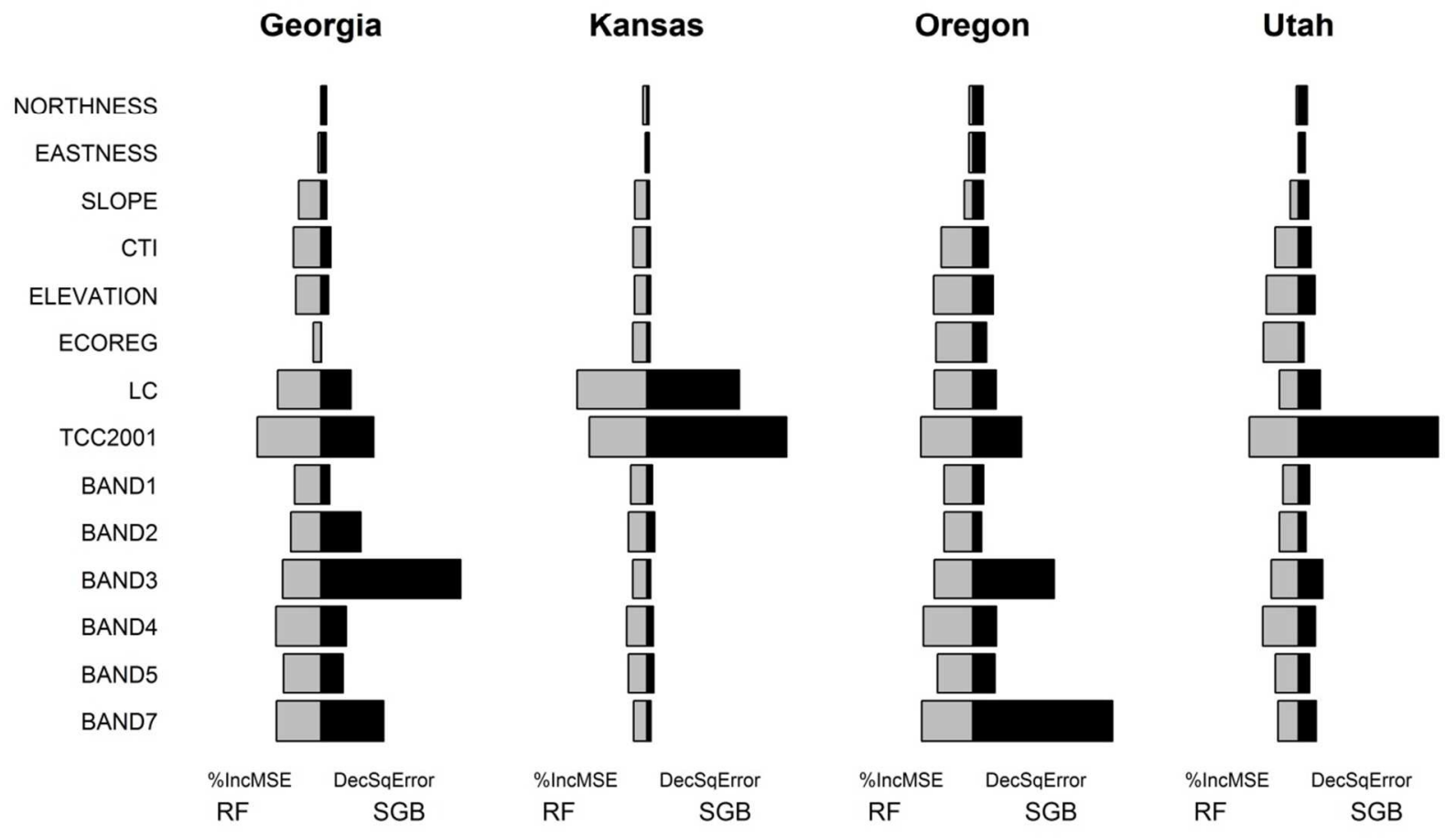

Figure 4 Variable importance for the RF and SGB models of the 4 pilot regions. RF on the left in gray, and SGB on the right in black. RF importance measured by the percent increase in MSE with random permutation of each variable. SGB importance measured by the decrease in squared error attributed to each variable in the gradient of the loss function. 


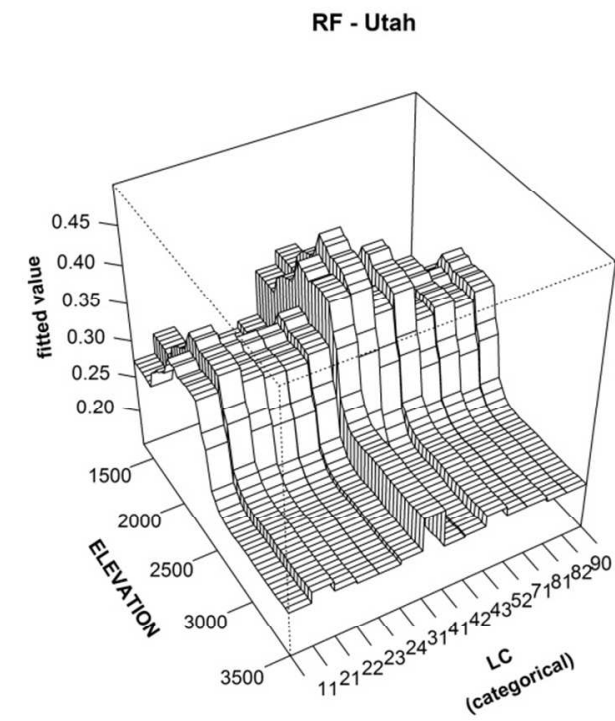

SGB - Utah

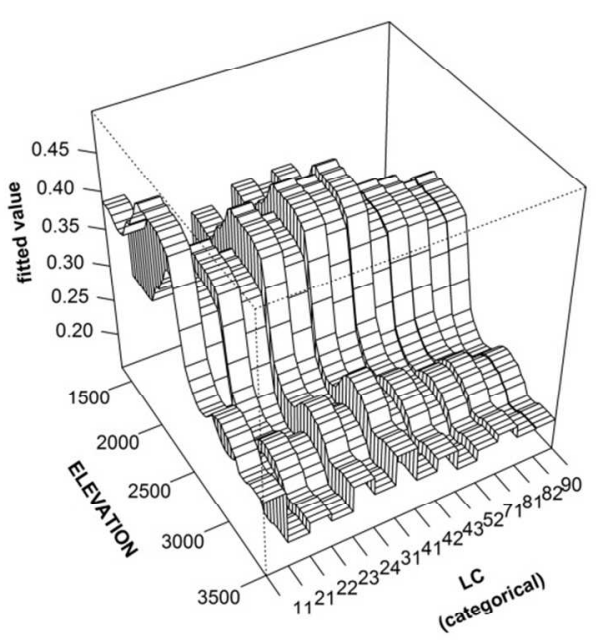

RF - Utah

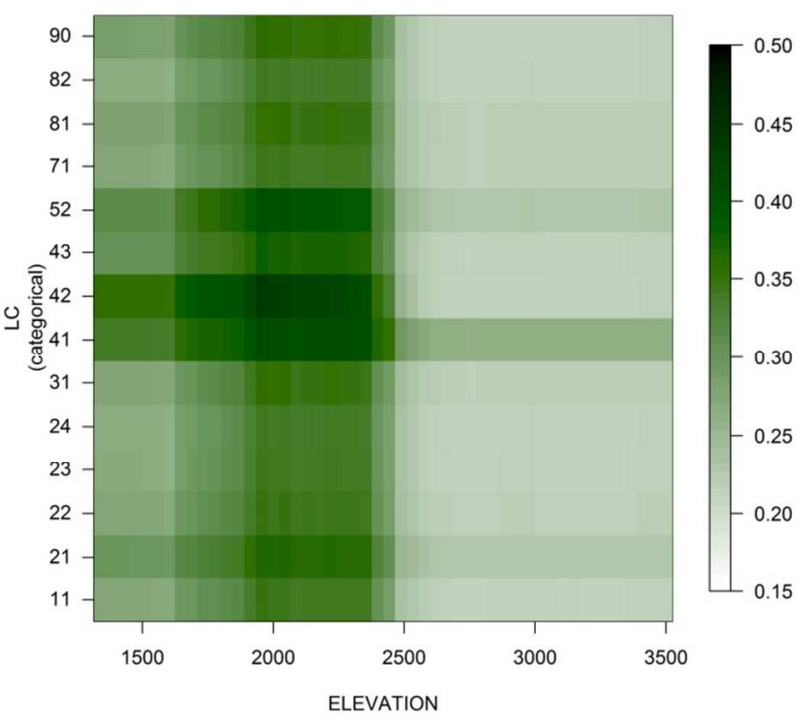

SGB - Utah

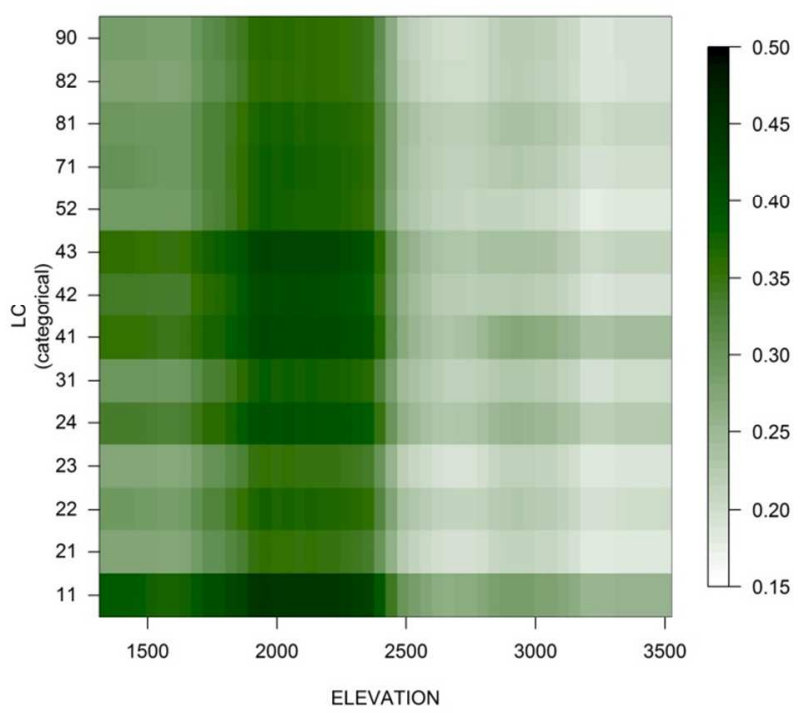

Figure 5 Interaction plots for elevation (ELEVATION) and land cover class (LC) for final Utah models. These figures show the effect of changes in two predictor variables on predicted TCC, with all other variables held at their mean (or majority). To see an example of interaction, look at the RF model, and the effect of elevation in land cover classes 41 (Deciduous Forest) and 42 (Evergreen Forest). In class 41, TCC is moderate at the lowest elevations in the UT region, increases at mid elevations, and drops slightly to moderate levels at high elevations. In class 42, TCC starts slightly higher than class 41 at low elevations, again rises at mid elevations, but then drops to near zero at high elevations. Also notice the differences between the RF model and the SGB model. Even though overall accuracy of the two models is similar, the predicted TCC in Land Cover Class 11 (open water) is very different. 

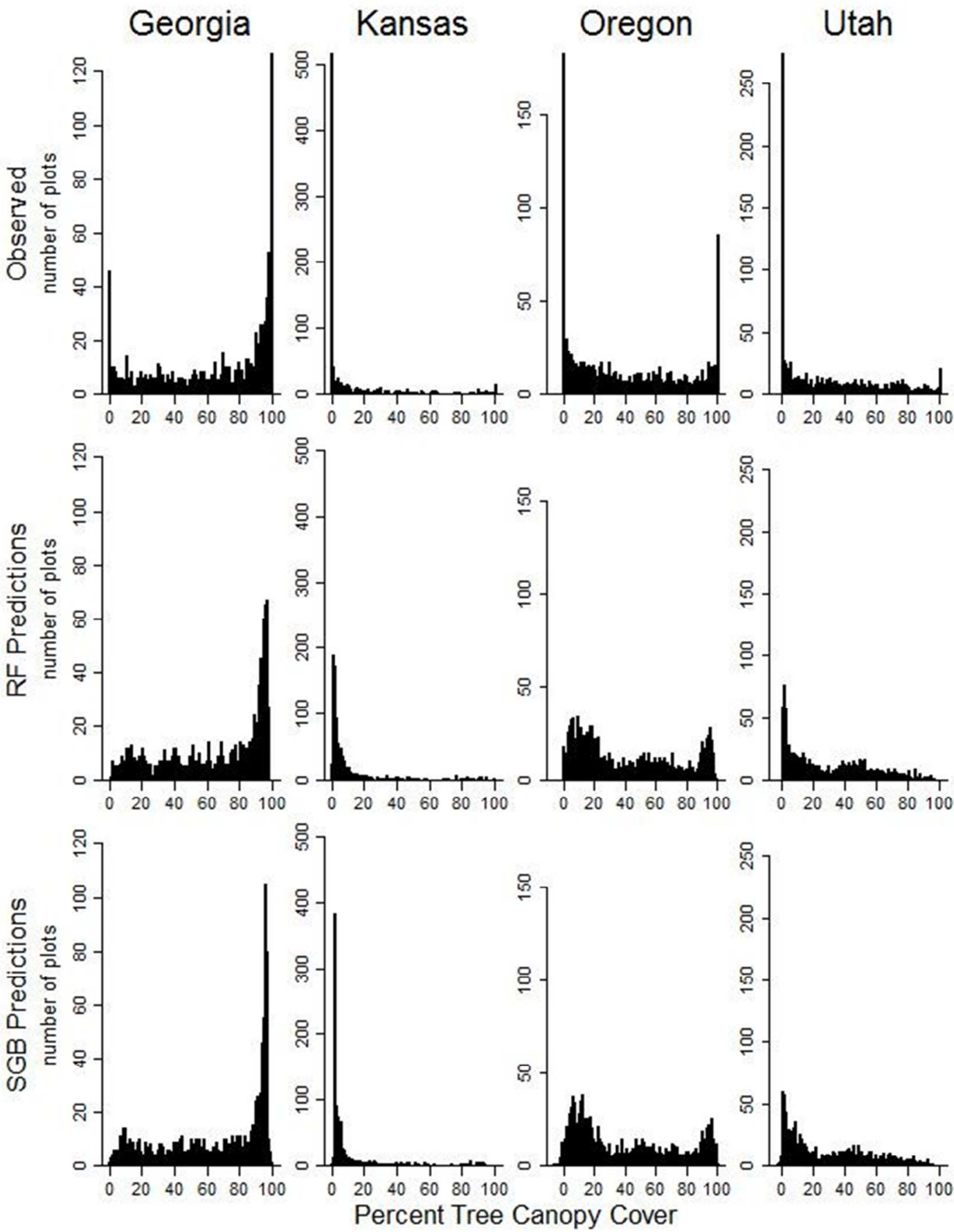

Percent Tree Canopy Cover

Figure 6 Histograms illustrating number of photo plots in the independent test sets by percent tree canopy cover (1\% classes). The observed plot distribution varies across the four regions. Georgia and Oregon include plots across the full range of possible canopy cover, with spikes in the number of plots at $0 \%$ and $100 \%$. Utah is similar, except that it has a higher proportion of low canopy cover plots, and fewer plots with $100 \%$ canopy cover. In Kansas, the majority of the plots have very low canopy cover, with only a small number of plots at higher canopy covers. Both RF and SGB models had difficulty capturing the observed spikes in number of plots at $0 \%$ and $100 \%$. 

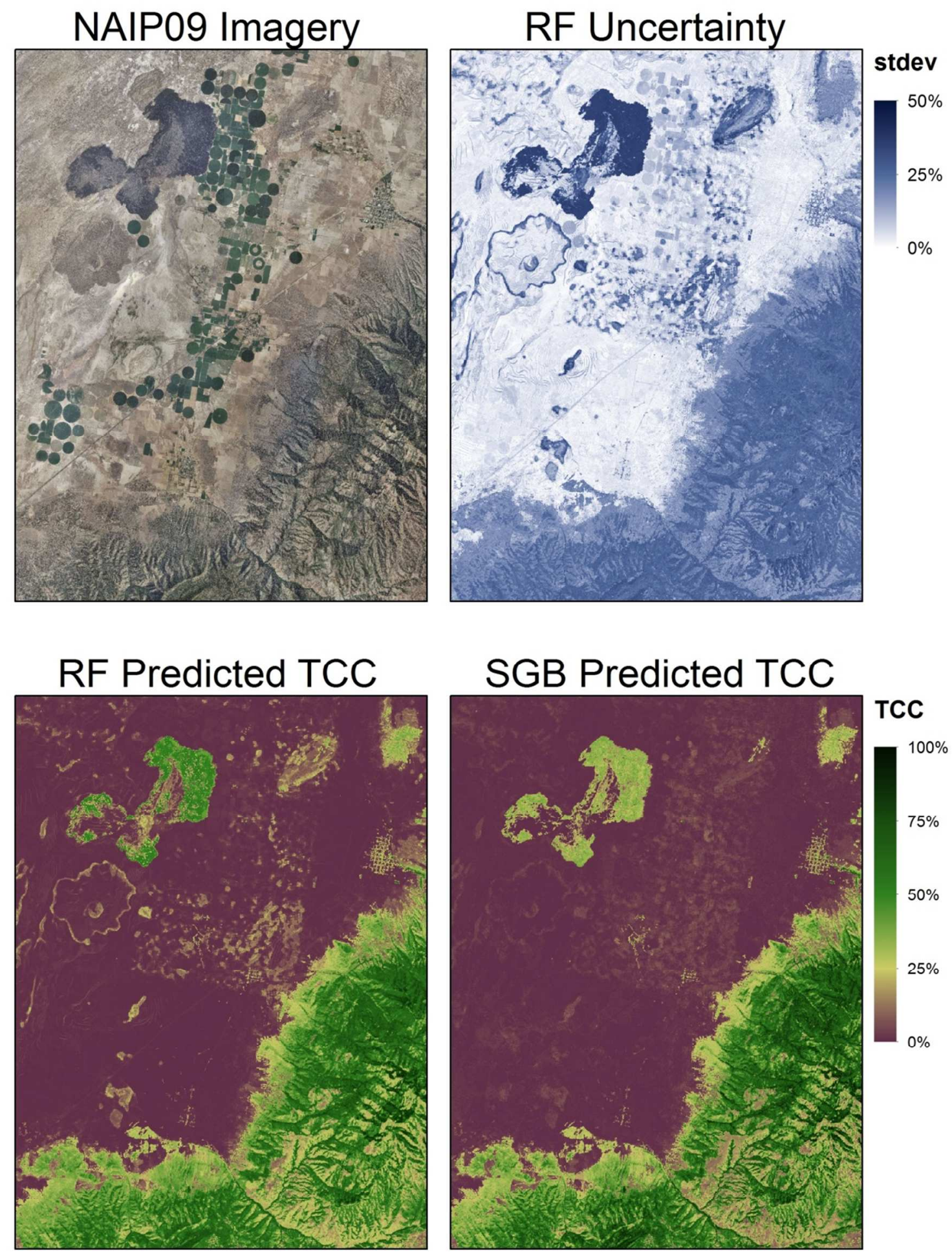

Figure 7 Detail map of northwest portion of the Utah region, showing NAIP09 imagery, The RF Uncertainty map, and Random Forest and Stochastic Gradient Boosting predictions for Tree Canopy Cover. The dark patch in the upper left of the photo is a lava bed. Both RF and SGB mistook it for an area of moderate TCC (RF predicted slightly higher cover than SGB). The RF uncertainty map shows that while the mean prediction from the $\mathbf{1 5 0 0}$ trees in the model was for moderate TCC, there was a very high level of uncertainty in these predictions, with some trees predicting low TCC while other trees predicting much higher TCC. 

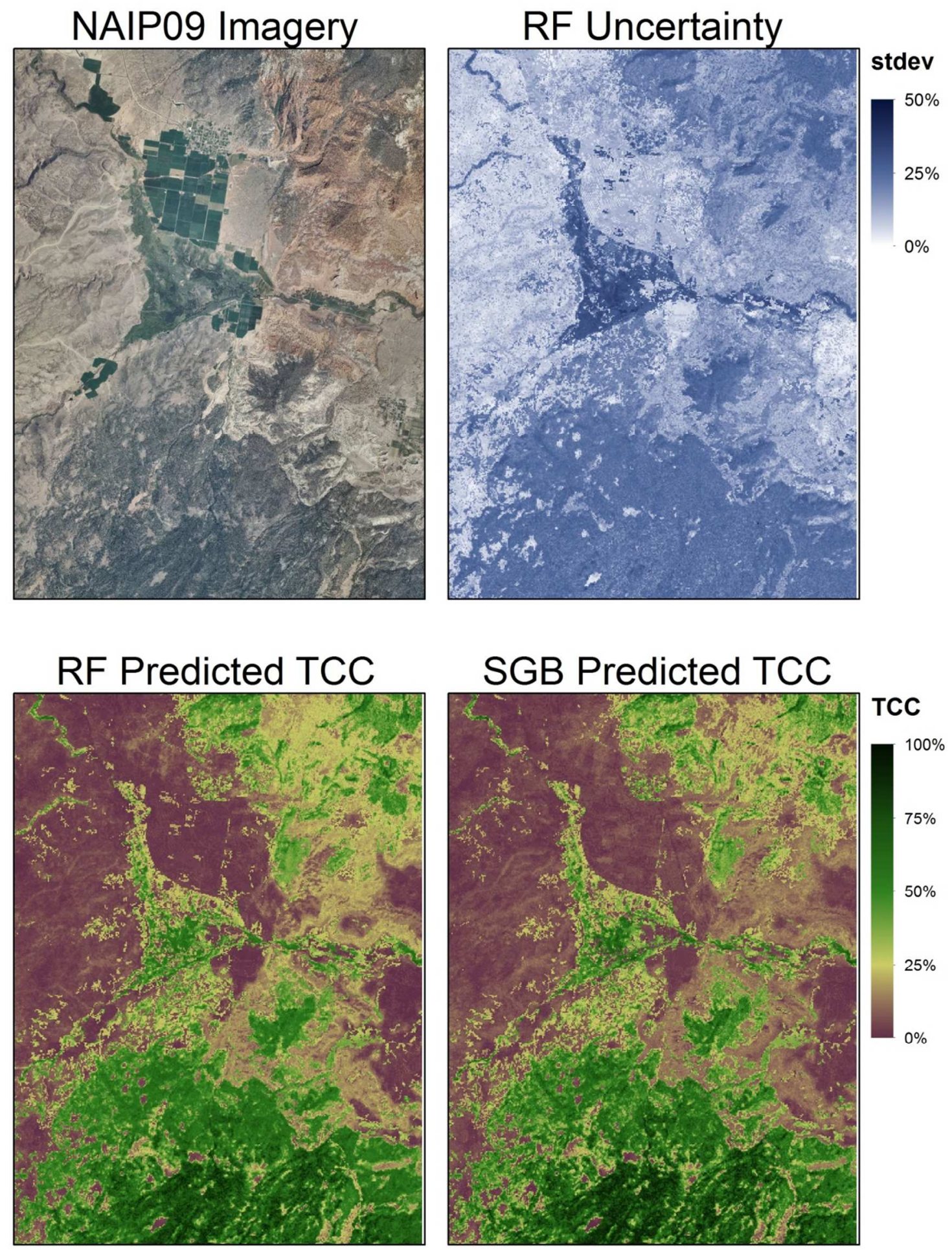

1016

1018

1019

1020

1021

1022

1023
Figure 8 Detail map of eastern portion of the Utah region, showing NAIP09 imagery, The RF Uncertainty map, and Random Forest and Stochastic Gradient Boosting predictions for Tree Canopy Cover. The dark triangular patch in the middle of the photo is a wetland. Both RF and SGB mistook it for an area of moderate to high TCC. The RF uncertainty map shows that while the mean prediction from the 1500 trees in the model was for moderate to high TCC, there was a very high level of uncertainty in these predictions, with some trees predicting low TCC while other trees predicting much higher TCC. The adjoining agricultural area was correctly identified as low TCC by both models, most likely due to the TCC2001 and the LC predictor layers. 